\title{
Management of ischaemia with non-obstructive coronary arteries (INOCA)
}

\author{
John F Beltrame, ${ }^{1,2,3}$ Rosanna Tavella, ${ }^{1,2,3}$ Dione Jones, ${ }^{1,2}$ Chris Zeitz $^{1,2,3}$
}

\author{
A) Check for updates \\ ${ }^{1}$ Adelaide Medical School, \\ University of Adelaide, Queen \\ Elizabeth Hospital Campus, \\ Woodville South, Adelaide, SA, \\ Australia \\ ${ }^{2}$ Department of Cardiology, \\ Central Adelaide Local Health \\ Network, SA Health, Adelaide, \\ SA, Australia \\ ${ }^{3}$ Basil Hetzel Institute for \\ Translational Health Research, \\ Queen Elizabeth Hospital, \\ Woodville South, Adelaide, SA, \\ Australia \\ Correspondence to: \\ J F Beltrame \\ john.beltrame@adelaide.edu.au \\ Cite thisas: BMJ2021;375:e060602 \\ http://dx.doi.org/10.1136/ \\ bmj-2021-060602 \\ Series explanation: State of the \\ Art Reviews are commissioned \\ on the basis of their relevance \\ to academics and specialists \\ in the US and internationally. \\ For this reason they are written \\ predominantly by US authors.
}

\begin{abstract}
Up to half of patients undergoing elective coronary angiography for the investigation of chest pain do not present with evidence of obstructive coronary artery disease. These patients are often discharged with a diagnosis of non-cardiac chest pain, yet many could have an ischaemic basis for their symptoms. This type of ischaemic chest pain in the absence of obstructive coronary artery disease is referred to as INOCA (ischaemia with non-obstructive coronary arteries). This comprehensive review of INOCA management looks at why these patients require treatment, who requires treatment based on diagnostic evaluation, what clinical treatment targets should be considered, how to treat patients using a personalised medicine approach, when to initiate treatment, and where future research is progressing.
\end{abstract}

\section{Introduction}

Patients with symptoms and signs suggestive of ischaemic heart disease but found to have no obstructive coronary arteries on angiography (that is, no coronary lesions on angiography $\geq 50 \%$ ) should be diagnosed with INOCA (ischaemia with non-obstructive coronary arteries). ${ }^{1}$ However, these symptoms are often dismissed as non-cardiac chest pain, and patients are given no explanation to account for their symptoms or no targeted treatment to alleviate their pain. Building on the previous literature, this comprehensive review provides a holistic approach to the management of INOCA by looking at why these patients require treatment, who requires treatment based upon diagnostic evaluation, what clinical treatment targets should be considered, how to treat patients using a personalised medicine approach, when to initiate treatment, and where future research is progressing (summarised in fig 1).

\section{Sources and selection criteria}

A literature search of PubMed and Embase databases for studies published between January 1980 and December 2020 in the English language was undertaken for the health risks associated with INOCA, original papers characterising the various clinical forms of INOCA, clinical studies defining the pathophysiological mechanisms of INOCA, and clinical trials evaluating the efficacy of treatments. In assessing the health risks associated with INOCA, we searched PubMed for prognostic studies using the following terms: "vasospastic angina," "variant angina," "Prinzmetal angina," "syndrome X," "microvascular angina," "coronary slow flow phenomenon," “microvascular spasm," "INOCA," "angina" or "chest pain" and "normal coronary arteries," "non-obstructive coronary artery disease," or "normal angiography." These terms were also used to identify the original papers where the clinical entities were created. The pathophysiological mechanisms responsible for INOCA were determined from review papers, with reference back to the original studies. Finally, the therapeutic clinical trials were identified by a search of PubMed and Embase from January 1980 to December 2020 with the following keywords: "angina” or "chest pain" and "normal coronary arteries," "non-obstructive coronary artery disease," or "normal angiography." This review was supplemented by the authors' own personal reference collections. Only clinical trials that investigated the impact of treatments on patient symptoms or adverse events were included in this report. Eligible studies were included if they were published between January 1980 and December 2020; in the English language; able to quantitate outcome from treatment, reported as either related to patient symptoms/functioning or clinical events.

In addition to providing data supporting the rationale for treating patients with INOCA, this review provides a pragmatic, holistic, patient focused strategy for managing these patients. The review includes diagnostic considerations to identify the underlying mechanisms and therefore appropriate treatment targets, as well as suggestions for when treatment should be initiated. These suggestions are based on previous publications and discussions during professional society conferences.

\section{Epidemiology}

The prevalence of INOCA is difficult to quantitate because of the limited population datasets that have documented the presence of myocardial ischaemia in the absence of obstructive coronary artery disease. However, a comprehensive US dataset of almost 

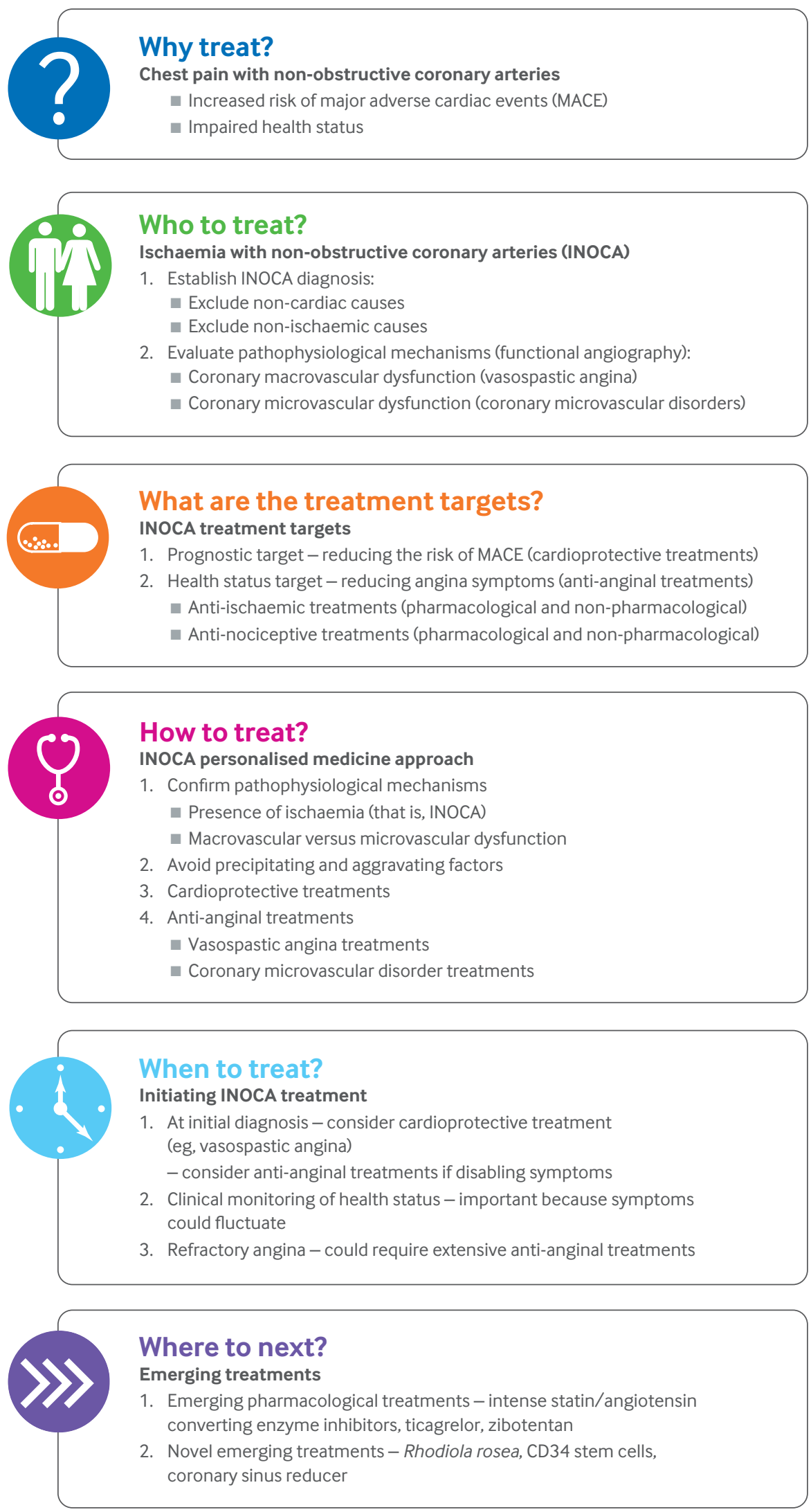

Fig 1 | Synopsis of clinical approaches for INOCA treatment 
400000 patients undergoing elective coronary angiography reported that $59 \%$ had no evidence of obstructive coronary artery disease. ${ }^{2}$ Although we do not know how many patients completely fulfilled the criteria for INOCA, the data suggest that more than half undergoing elective angiography could have INOCA. Moreover, smaller selective studies of INOCA have reported an increased risk of major adverse cardiovascular events (MACE), ${ }^{3}$ threefold to fourfold increased risk of hospital admission, ${ }^{4}$ and hence a high healthcare cost burden ${ }^{5}$ in these patients.

\section{Why treat?}

The treatment of cardiovascular disorders aims to prevent MACE or reduce symptoms to improve quality of life (that is, improve health outcomes or status). Thus, in order to justify the treatment of patients with INOCA, health professionals should understand the impact of the disorder on MACE and health status.

\section{Increased risk of MACE}

Prognostic studies of patients with INOCA have reported heterogeneous outcomes owing to different recruitment strategies, with some including an unselected patient cohort and others using more selective criteria such as documented ischaemia, coronary microvascular dysfunction, or vasospastic angina. Considering these limitations, a comprehensive meta-analysis reported an overall estimated incidence of MACE (all cause mortality and myocardial infarction) of 0.98 per 100 person years, which is less favourable than the 0.2 per 100 person years reported in a similarly aged general population in North America. ${ }^{3}$

Prospectively conducted international registries of vasospastic angina and microvascular angina have also provided insights into the incidence of MACE in patients with INOCA. A prospective, comprehensive, vasospastic angina registry in Japan showed a $6 \%$ frequency of MACE, ${ }^{6}$ with a subsequent extension study reporting that this proportion was slightly lower in Japanese patients than in white patients. ${ }^{7}$ Furthermore, a recent international registry of microvascular angina reported an annual MACE incidence of $7.7 \%$ per patient year, but found no ethnic differences in this outcome. ${ }^{8}$ Hence patients with INOCA have an under-appreciated risk of MACE and therefore warrant treatments to reduce MACE; however, no large prospective randomised clinical trials have been published that evaluate the benefit of cardioprotective treatments in these patients.

\section{Impaired health status}

Patients with chronic stable angina (irrespective of the presence or absence of obstructive coronary arteries) will have an impaired health status, where health status is defined as the impact of a disease on patient function as reported by the patient. ${ }^{9}$ This status includes the impact of angina symptoms, which produce functional limitations (eg, reduce exercise capacity), and how they affect quality of life (fig 2). ${ }^{10}$ Thus, reducing angina symptoms would be expected to improve physical functioning and quality of life.

An estimated $50 \%$ of patients with stable chest pain and normal angiography will experience recurrent episodes of chest pain, ${ }^{11}{ }^{12}$ which is a similar rate to those with obstructive coronary artery disease. $^{12}$ Furthermore, about $15-25 \%$ of these patients are readmitted with chest pain or undergo repeat angiography (or both), thereby also affecting health resources. ${ }^{4}$ Moreover, compared with healthy controls, patients with chest pain and nonobstructive coronary arteries have a reduced quality of life at 12 months. ${ }^{12}$

Thus, patients with INOCA have both a small increased risk of MACE and significantly impaired health status, thereby underscoring the importance of considering both cardioprotective treatments to reduce MACE and anti-anginal treatments to improve symptoms and health status.

\section{Who to treat?}

The decision on who to treat is based on two fundamental diagnostic considerations: (1) is the chest pain or presenting symptom ischaemic in nature (that is, INOCA), and (2) what is the underlying pathophysiological mechanisms responsible for the ischaemic symptom (fig 1)? Answering these questions will identify a cohort of patients with INOCA who could benefit from pathophysiologically targeted treatments.

INOCA is common, is under-diagnosed, requires careful assessment to exclude non-ischaemic causes, and often needs functional coronary angiography to identify the underlying vasomotor pathophysiological mechanism responsible for the chest pain. Although macrovascular coronary spasm (vasospastic angina) is well accepted because it can be visualised on angiography, coronary microvascular disorders remain more elusive owing to multiple potential mechanisms.

\section{Identification of INOCA in patients}

Owing to the existence of multiple causes of chest pain, diagnostic evaluation of patients with chest pain and non-obstructive coronary arteries requires excluding non-cardiac and non-ischaemic causes, and obtaining clinical evidence implicating the presence of myocardial ischaemia.

Figure 3 summarises common non-cardiac and non-ischaemic causes of chest pain in the absence of obstructive coronary arteries on angiography. These causes can often be delineated with a comprehensive clinical history but might need additional investigations such as endoscopy, oesophageal manometry, biliary ultrasonography, and echocardiography. Ideally, these causes should have been excluded before invasive angiography, thereby avoiding this invasive procedure. Moreover, these disorders should be excluded before embarking on cardiac treatments, although occasionally a trial of anti-anginal treatment might be warranted when the underlying cause remains unclear. 


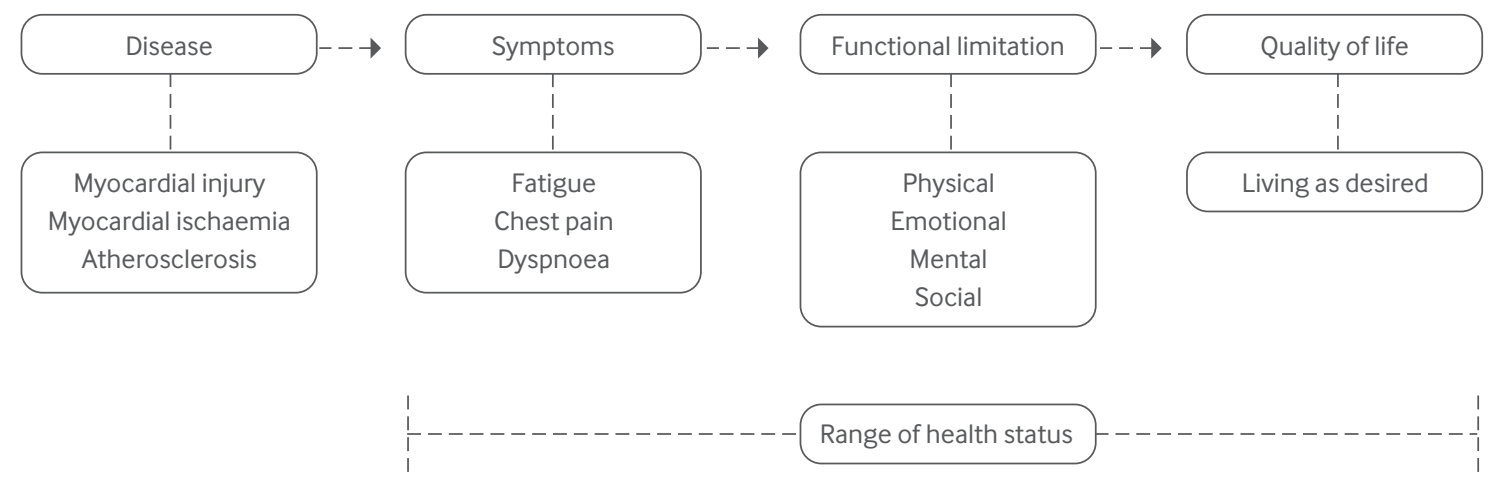

Fig 2 | Overview of health status domains in coronary artery disease. Adapted with permission from Spertus $(2008)^{10}$

After excluding non-cardiac or non-ischaemic causes of the chest pain, evidence of myocardial ischaemia should be ascertained to make the diagnosis of INOCA. The term "myocardial ischaemia” describes an inadequate coronary blood flow to supply the myocardium's metabolic demand, so that myocardial cells become hypoxic and accumulate lactic acid. This pathophysiological concept requires the measurement of biochemical markers, but these are seldom measured in routine clinical practice. Thus, clinical surrogates are used as markers of myocardial ischaemia including:

- Typical angina pectoris that might occur with exertion, stress, or at rest (that is, a strangling sensation in the chest-from the Greek word "ankhone" and Latin word "pectoris")

- Ischaemic ECG (electrocardiogram) changes
- Impaired myocardial perfusion on stress imaging

- $\quad$ Stress induced regional wall motion abnormality

- Abnormal hyperaemic coronary blood flow responses (that is, impaired coronary flow reserve).

When one of these markers is documented, the patient is considered as having ischaemic chest pain, and thus a diagnosis of INOCA would be considered in the absence of obstructive coronary artery disease (that is, no coronary lesions $\geq 50 \%$ on angiography).

\section{Pathophysiological mechanisms responsible for INOCA}

The potential pathophysiological mechanisms responsible for myocardial ischaemia could include an impaired coronary blood flow (epicardial coronary artery spasm or coronary microvascular dysfunction)

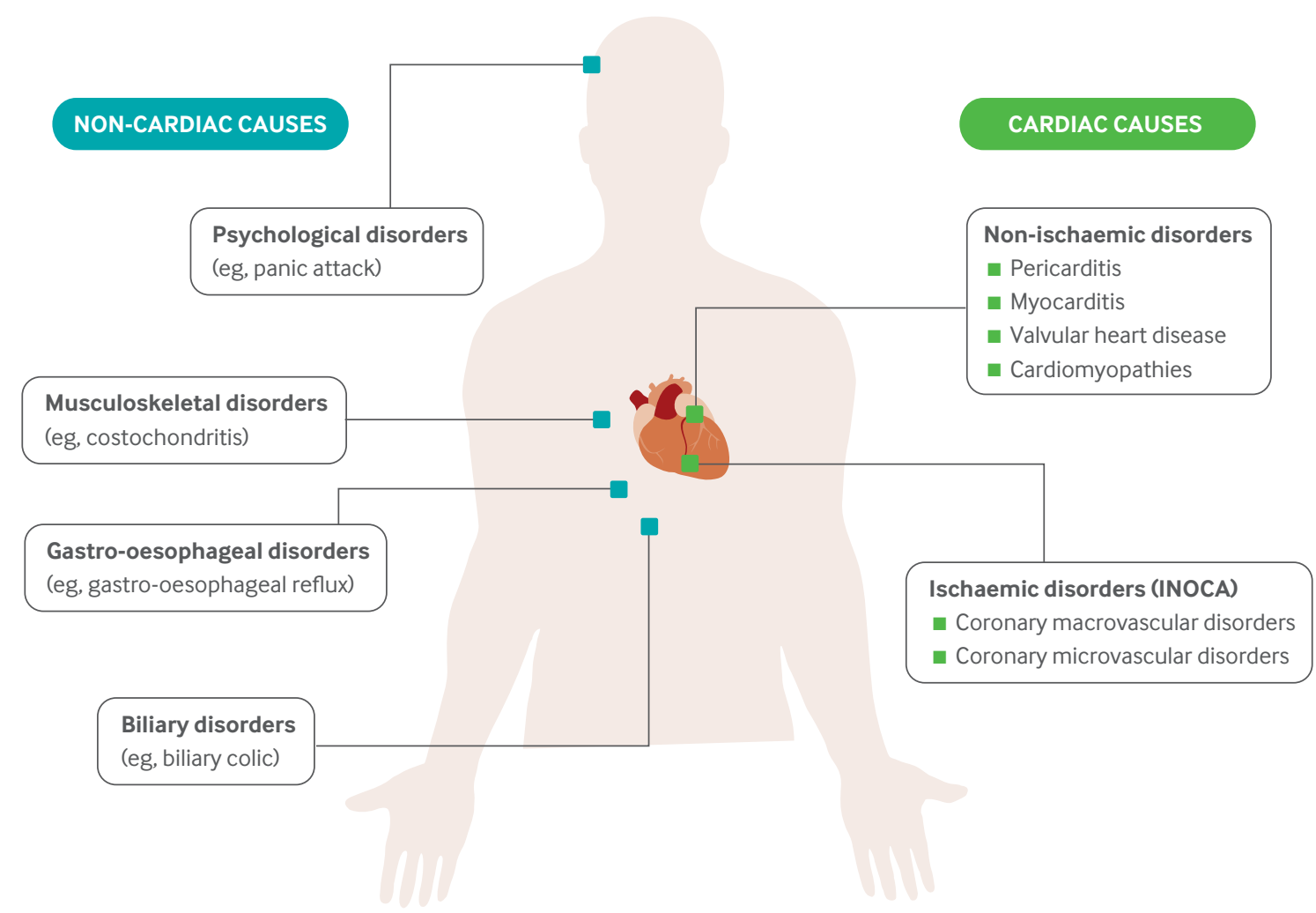

Fig 3 | Common causes of chest pain in patients with non-obstructive coronary arteries. INOCA=ischaemia with non-obstructive coronary arteries 
or increased myocardial oxygen demand (that is, tachycardia or myocardial workload). These mechanisms might be evident during the clinical presentation, for example, a spontaneous episode of coronary artery spasm during diagnostic angiography or a tachyarrhythmia at 170 beats per minute during chest pain. However, often the underlying mechanism is not evident on initial evaluation and further assessment beyond conventional invasive structural coronary angiography is required.

\section{Use of functional coronary angiography to identify} underlying mechanisms

In structural coronary angiography, the luminal coronary anatomy is imaged, and the presence of obstructive coronary artery disease (usually due to atherosclerotic disease) evaluated as a cause of the chest pain. In functional coronary angiography, pathophysiological assessment of the coronary circulation is undertaken to assess:

- Whether equivocal coronary stenoses are functionally obstructive, via measurement of fractional flow reserve

- Whether coronary microvascular dysfunction is present by evaluation of coronary flow reserve (CFR), microvascular resistance measures (index of microvascular resistance or hyperaemic microvascular resistance), or the presence of the angiographic coronary slow flow phenomenon

- Whether inducible coronary artery spasm is present on provocative spasm testing with intracoronary acetylcholine.

Although functional coronary angiography identifies key potential pathophysiological mechanisms responsible for ischaemic chest pain in INOCA, it is rarely conducted in the large cohort of patients with unexplained chest pain and nonobstructive coronary arteries.

The CorMICA trial randomised 151 patients with angina and non-obstructive coronary artery disease to either functional angiography with a stratified medical treatment plan based on investigation findings (that is, spasm or coronary microvascular dysfunction) or standard care. Patients in the intervention arm showed improved angina status on the Seattle Angina Questionnaire summary score at six months (11.68 points, 95\% confidence interval 4.99 to $18.37 ; \mathrm{P}=0.001$ ) and at one year (13.6 points; 7.3 to 19.9 ; $\mathrm{P}<0.001) .{ }^{13}{ }^{14}$ The use of functional coronary angiography is further evident from other reports, ${ }^{1516}$ where $80-90 \%$ of patients evaluated have had abnormal coronary haemodynamic findings, thereby providing explanations for their chest pain. Considering the clinical use of functional coronary angiography, its techniques have been recommended in professional society guidelines ${ }^{1} 7$

\section{Coronary macrovascular functional disorders (coronary artery spasm)}

More than 60 years ago, Prinzmetal described 32 cases of "variant angina," with features that differed from the classic angina detailed by Heberden ${ }^{18}$ because the pain occurred at rest rather than being precipitated by exertion, and it was often associated with transient ST elevation rather than ST depression. ${ }^{19}$ Since this first description, improved understanding of the syndrome has evolved with the advent of coronary angiography confirming that epicardial coronary artery spasm was responsible for the syndrome. More recently, the disorder has been referred to as "vasospastic angina" with clinical diagnostic criteria including nitrate responsive angina, transient ischaemic ECG changes (either ST elevation or ST depression), and documented coronary artery spasm. ${ }^{20}$ Although a spontaneous witnessed episode might confirm the diagnosis, provocative spasm testing during functional coronary angiography is often required. This testing involves incremental intracoronary doses of acetylcholine with coronary artery spasm confirmed on angiography. The importance of diagnosing vasospastic angina is its associated morbidity and mortality. Patients might experience recurrent disabling chest pain and could even develop acute myocardial infarction or sudden cardiac death. ${ }^{21}$ These adverse events could be prevented by effective treatment, so the diagnosis of vasospastic angina is imperative in the evaluation of patients with INOCA.

\section{Coronary microvascular disorders}

Coronary microvascular disorders are a frequent cause of INOCA but are less well understood and more complex than vasospastic angina; however, the two conditionsmight coexist, adding further complexity to the clinical picture. Vasospastic angina arises from exaggerated coronary artery constrictor response (attributable to vascular smooth muscle hyperreactivity). ${ }^{22}$ By contrast, multiple mechanisms might be responsible for coronary microvascular disorders ${ }^{24}$ suchasanincreased resting microvascular tone, microvascular spasm ${ }^{25}$ (or hyper-reactivity), or a failure of the microvasculature to dilate in response to an increased myocardial oxygen demand. ${ }^{26}$ These heterogeneous pathophysiological mechanisms have formed the basis of several coronary microvascular disorders, as summarised in table 1.

Cardiac syndrome $X$-this syndrome was one of the first coronary microvascular disorders described, ${ }^{28}$ and initially specifically included patients with exertional chest pain, ischaemic ST depression on exercise stress testing, an absence of obstructive coronary artery disease, and no evidence of coronary artery spasm. It attracted considerable interest and important data over the past 30 years, but the term "cardiac syndrome X" has been progressively misused to describe any patient with INOCA. This confusion in the nomenclature and negative connotations for patients has prompted the avoidance of this diagnostic term; however, we have included it in this review because important clinical trials have been conducted in patients with specific criteria for the syndrome.

Microvascular angina-this term was initially used to describe patients who had chest pain 


\begin{tabular}{|c|c|c|c|c|}
\hline & Diagnostic investigation & Putative mechanism & Clinical presentation & $\begin{array}{l}\text { Nitrate } \\
\text { response }\end{array}$ \\
\hline \multicolumn{5}{|c|}{ Coronary macrovascular disorders } \\
\hline Vasospastic angina $^{27}$ & Spontaneous or inducible coronary artery spasm & Coronary artery spasm & $\begin{array}{l}\text { Rest or unstable angina; smoking is a risk } \\
\text { factor }\end{array}$ & Prompt \\
\hline \multicolumn{5}{|c|}{ Coronary microvascular disorders ${ }^{24}$} \\
\hline Cardiac syndrome $\mathrm{X}$ & Positive stress ECG & Impaired microvascular vasodilation & $\begin{array}{l}\text { Stable exertional angina; high prevalence in } \\
\text { female patients }\end{array}$ & Limited \\
\hline Microvascular angina & Impaired coronary flow reserve & Impaired microvascular vasodilation & Often rest angina; often female patients & Limited \\
\hline $\begin{array}{l}\text { Coronary slow flow } \\
\text { phenomenon }\end{array}$ & Delayed vessel opacification on angiography & Increased microvascular resistance & Unstable angina; smoking is a risk factor & Variable \\
\hline Microvascular spasm & $\begin{array}{l}\text { Inducible pain and ischaemic ECG changes with } \\
\text { acetylcholine, but no coronary artery spasm }\end{array}$ & Microvascular spasm & Unstable angina; often female patients & Variable \\
\hline
\end{tabular}

with an impaired coronary flow reserve (that is, less than a doubling of the coronary blood flow response to a standard hyperaemic stimulus) in the absence of obstructive coronary artery disease. ${ }^{29}$ More recently, it has become the generic term for patients with a primary coronary microvascular disorder characterised by ischaemic chest pain, non-obstructive coronary arteries on angiography, evidence of myocardial ischaemia, and evidence of coronary microvascular dysfunction (eg, abnormal coronary flow reserve). ${ }^{30}$

Coronary slow flow phenomenon-this event is an angiographic phenomenon characterised by the delayed passage of angiographic contrast in the absence of obstructive coronary arteries. ${ }^{31}$ This coronary microvascular disorder was the first to raise awareness that patients with coronary microvascular disorders might initially present with unstable angina, rather than a chronic stable angina presentation. $^{32}$

Microvascular spasm-this disorder is diagnosed when intracoronary acetylcholine during functional coronary angiography precipitates chest pain and ischaemic ECG changes but not epicardial coronary artery spasm, in patients without obstructive coronary artery disease. ${ }^{33}$ Therefore, this coronary microvascular disorder is readily diagnosed during acetylcholine provocation testing, although its association with vasospastic angina is difficult to define.

\section{What are the treatment targets?}

Patients with INOCA have an increased risk of MACE and impaired health status, so treatment should be targeted at improving prognosis by preventing MACE, and improving quality of life by optimising symptom control (fig 1).

\section{Prognostic target-reducing the risk of MACE}

Among patients with INOCA, MACE has considerable heterogeneity, with a higher incidence in those individuals who have had typical angina or mild-tomoderate atherosclerotic disease on angiography. ${ }^{3}$ This heterogeneity in outcomes reflects the variation in the INOCA cohorts studied, since not all patients undergo comprehensive functional coronary angiography to delineate the multiple pathophysiological mechanisms responsible for the vasomotor disorders.

\section{Vasospastic angina}

Vasospastic angina has long been associated with a risk of MACE, including acute myocardial infarction, malignant ventricular arrhythmias, and sudden cardiac death. ${ }^{21}$ Indeed, the Japanese Coronary Spasm Association has validated a risk score for predicting MACE in patients with vasospastic angina, with key clinical determinants including out-ofhospital cardiac arrest, obstructive coronary artery disease, rest angina, smoking, multivessel spasm, ST elevation during a spontaneous angina attack, and beta blocker use. ${ }^{6}$ Therefore, the prevention of MACE in vasospastic angina requires these risk factors to be managed (that is, cardiac arrest, coronary artery disease, smoking, and beta blocker use).

\section{Coronary microvascular disorders}

Coronary microvascular disorders provide a greater dilemma in targeting cardioprotective treatment to reduce MACE. Firstly, the increased risk of MACE in these disorders appears small, with some studies providing conflicting results. Secondly, the heterogenous endotypes within this form of INOCA could differ in their risk of MACE and thus in their requirement for cardioprotective treatment. Finally, because functional coronary angiography is not done routinely, pathophysiological characteristics of the responsible underlying mechanism might be missed in many patients with INOCA. These compounding issues highlight the ongoing problems with identifying cardioprotective agents for these disorders.

\section{Health status target-reducing angina symptoms to} improve quality of life

In a novel investigation, the health status of patients with suspected INOCA were compared with those who had obstructive coronary artery disease associated with stable angina and with healthy controls. ${ }^{12}$ The angina frequency, physical limitation, and quality of life in the 12 months after elective angiography for the investigation of chest pain was similar irrespective of the presence or absence of obstructive coronary artery disease (fig 4). Moreover, the only predictor of 
ongoing chest pain at 12 months was female sex. ${ }^{12}$ Furthermore, both physical and mental quality of life indices at 12 month follow-up were worse in patients with INOCA than in healthy controls with no history of chest pain. ${ }^{12}$ Thus, improving health status is key in the treatment for INOCA, which should target improving angina symptoms since the frequency of angina is related to quality of life. ${ }^{34}$

Targeting the control of angina symptoms can involve two approaches: an anti-ischaemic approach or an anti-nociceptive approach (table 2 ). Because the underlying mechanism responsible for the angina symptom is primarily believed to be ischaemic in nature, the anti-ischaemic approach to the underlying pathophysiology should be first considered; but in patients with chronic chest pain syndromes, the anti-nociceptive approach might also need to be used.

\section{Anti-ischaemic approach}

Pharmacological anti-ischaemic treatments

Pharmacological anti-ischaemic treatments can prevent or reduce ischaemia and thus improve symptoms. Conventional anti-ischaemic agents established in patients with stable angina and with obstructive coronary artery disease have also shown variable effectiveness in INOCA. These agents include long acting nitrates, ${ }^{35} 36$ beta blockers, ${ }^{37}$ calcium channel blockers, ${ }^{37}{ }^{38}$ ivabradine, ${ }^{39}$ nicorandil, ${ }^{40-42}$ ranolazine, $^{38} 39$ perhexiline, ${ }^{43}$ and trimetazidine $^{44}$ (tables 2, 3, and 4). They can achieve their anti-ischaemic effects by reducing myocardial oxygen demand (thereby reducing heart rate, blood pressure, cardiac contractility, or altering myocardial metabolism), or increasing coronary blood supply (by reducing contraction vascular smooth muscle cell and thus coronary tone). These anti-ischaemic agents

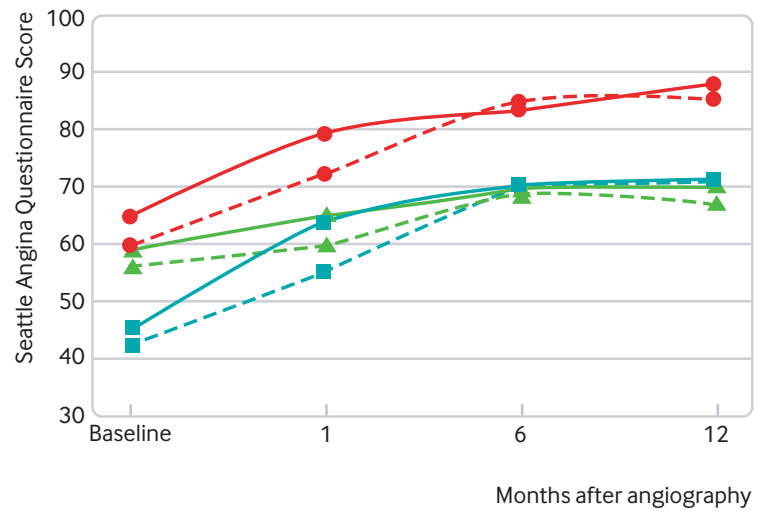

$$
\begin{aligned}
& \text { Frequency of anginal episodes } \\
& -A-C A D-\text { Physical limitation } \\
& - \text { - CAD - Angina frequency } \\
& - \text { - INOCA - PAD - Quality of life }
\end{aligned}
$$

Fig 4 | Health status over 12 months in patients investigated for chest pain with or without obstructive coronary artery disease. $C A D=$ coronary artery disease; INOCA=ischaemia with non-obstructive coronary arteries. Adapted with permission from Tavella et al $(2016)^{12}$ achieve their therapeutic effects via different targets (table 2) and hence are often used in combination. In addition to these conventional agents, novel anti-ischaemic agents have been shown to reduce ischaemia or angina in patients with INOCA including phosphodiesterase- $3^{57}$ and phosphodiesterase $-5^{76}$ inhibitors, rho kinase inhibitors, ${ }^{96} 97$ angiotensin converting enzyme inhibitors ${ }^{72}$ and estradiol, ${ }^{54}$ as well as lifestyle risk factor modification ${ }^{87} 91$ (tables 3 and 4).

\section{Non-pharmacological anti-ischaemic treatments}

Non-pharmacological anti-ischaemic treatments of potential use are summarised in table 2. Exercise training has been shown to be beneficial in patients with INOCA, with the proposed mechanism being an improvement in endothelial function. ${ }^{98}$ Stellate ganglion blockade or thoracic sympathectomy has been shown to reduce symptoms and ischaemic ECG changes in patients with vasospastic angina, ${ }^{99}$ possibly by interrupting the influence of cardiac sympathetic efferent pathways on coronary vessels. However, whether this cardiac sympathectomy approach reduces symptoms by inhibiting coronary spasm or disrupting sympathetic pain afferents (that is, the nociceptive effect) remains unclear. Other anti-ischaemic devices that have been reported to improve symptoms in patients with coronary microvascular disorders include enhanced external counterpulsation ${ }^{100}$ and the coronary sinus reducer $^{101}$ (table 2 ).

\section{Anti-nociceptive approach}

Patients with coronary microvascular disorders have shown hyperalgesic responses to pain, irrespective of the presence of ischaemia. ${ }^{102}$ The hyperalgesic nociceptive pathway could arise from abnormalities at the level of pain receptors (adenosine might be a nociceptive stimulus), afferent sympathetic pain fibres, or unregulated pain transmission through the thalamus to the cortex..$^{102}$ Accordingly, therapeutic strategies that modulate the pain pathway-by inhibiting nociceptive adenosine receptors with methylxanthines, ${ }^{103}$ inhibiting afferent pain neurones with tricyclic antidepressants ${ }^{104}$ or neurostimulators, ${ }^{105}$ blocking pain transmission at the spinal cord level with analgesics or spinal cord stimulators, ${ }^{106}$ or modulating cortical inputs with cognitive behaviour therapy ${ }^{107}$-have been used in refractory angina. Therefore, this approach provides an alternative strategy to managing angina, particularly when it is refractory to anti-ischaemic treatments.

\section{How to treat?}

The above treatment targets for INOCA (that is, reducing the risk of MACE with cardioprotective agents and improving symptoms with anti-anginal treatments) need to be applied in the context of knowing who we are treating for INOCA (that is, those people with ischaemic chest pain due to coronary macrovascular spasm or microvascular dysfunction), so that the question of how to treat 


\begin{tabular}{|c|c|}
\hline Anti-anginal treatment & Possible mechanisms and clinical effect \\
\hline \multicolumn{2}{|l|}{ Anti-ischaemic targets } \\
\hline \multicolumn{2}{|l|}{ Anti-ischaemic pharmacological treatments } \\
\hline Beta blockers (eg, atenolol, metoprolol) & Inhibit myocardial beta 1 receptors (to reduce heart rate and contractility) \\
\hline $\begin{array}{l}\text { Calcium channel blockers, non-dihydropyridine (eg, } \\
\text { verapamil, diltiazem) }\end{array}$ & $\begin{array}{l}\text { Inhibit L calcium channels in myocardial cells to reduce heart rate and contractility in specialised } \\
\text { conducting tissue to reduce heart and in VSMCs leading to vasodilation }\end{array}$ \\
\hline $\begin{array}{l}\text { Calcium channel blockers, dihydropyridine (eg nifedipine, } \\
\text { amlodipine) }\end{array}$ & Inhibit L calcium channels in VSMCs (leading to vasodilation) \\
\hline $\begin{array}{l}\text { Long acting nitrates (eg, isosorbide dinitrate, isosorbide } \\
\text { mononitrate) }\end{array}$ & $\begin{array}{l}\text { Increase nitric oxide release (to increase cGMP levels in VSMCS, leading to vasodilation); more potent } \\
\text { systemic venodilator (reducing preload) than arterial dilator; epicardial coronary vasodilator }\end{array}$ \\
\hline Potassium channel activator (eg, nicorandil) & $\begin{array}{l}\text { Open VSMC potassium ATP channels (leading to vasodilation); increase nitric oxide release (to increase } \\
\text { cGMP levels in VSMCs, leading to vasodilation); balanced vasodilator in veins and arteries; epicardial and } \\
\text { microvascular coronary vasodilator }\end{array}$ \\
\hline Late sodium current blockers (eg, ranolazine) & $\begin{array}{l}\text { Inhibit myocyte late sodium currents (to reduce sodium dependent calcium overload); believed to } \\
\text { improve myocardial relaxation (thus reducing myocardial oxygen demand and improving microvascular } \\
\text { perfusion) }\end{array}$ \\
\hline Fatty acid oxidation inhibitors (eg, trimetazidine) & $\begin{array}{l}\text { Shift cardiac metabolism from fatty acid oxidation to glucose oxidation (to reduce myocardial oxygen } \\
\text { demand) }\end{array}$ \\
\hline Sinus node inhibitors (eg, ivabradine) & Inhibit sinoatrial node $I_{\mathrm{f}}$ current (to reduce heart rate) \\
\hline Angiotensin converting enzyme inhibitors (eg, enalapril) & Inhibit conversion of angiotensin I to II (leading to vasodilation) \\
\hline Phosphodiesterase-3 inhibitors (eg, cilostazol) & Increase CAMP levels in VSMCs (leading to vasodilation) \\
\hline Phosphodiesterase-5 inhibitors (eg, sildenafil) & $\begin{array}{l}\text { Increase cGMP levels in VSMCs (leading to vasodilation); caution with nitrates owing to increased cGMP } \\
\text { concentrations, which can lead to hypotension }\end{array}$ \\
\hline Rho kinase inhibitors (eg, fasudil) & Inhibit VSMC rho kinase (to increase MLC dephosphorylation, leading to vasodilation) \\
\hline Statins (eg, pravastatin, fluvastatin) & $\begin{array}{l}\text { Increase endothelial nitric oxide release (to increase cGMP levels in VSMCs, leading to vasodilation); } \\
\text { inhibit rho kinase pathway in VSMCs (to increase MLC dephosphorylation, leading to vasodilation) }\end{array}$ \\
\hline \multicolumn{2}{|l|}{ Anti-ischaemic non-pharmacological treatments } \\
\hline Stellate ganglion block & $\begin{array}{l}\text { Reduces cardiac sympathetic tone (to reduce vasoconstriction); disrupts cardiac sympathetic pain } \\
\text { afferent pathway }\end{array}$ \\
\hline External counterpulsation & $\begin{array}{l}\text { Promotes retrograde aortic diastolic coronary flow (to increase coronary perfusion); reduces left } \\
\text { ventricular diastolic pressure (to reduce myocardial oxygen demand); increases blood flow (leading to } \\
\text { endothelial homeostasis and increased endothelial function) }\end{array}$ \\
\hline Coronary sinus reducer & Increases coronary venous pressure (leading to arteriolar dilation and increased perfusion) \\
\hline \multicolumn{2}{|l|}{ Anti-nociceptive targets } \\
\hline Tricyclic antidepressants (eg, imipramine) & Block noradrenaline reuptake (to inhibit pain modulating neurons, and reduce pain) \\
\hline Methylxanthines (eg, theophylline) & Inhibit adenosine $\mathrm{A}_{2 \mathrm{~B}}$ receptor (to reduce pain) \\
\hline Opiates & Activate dorsal root ganglion and peripheral nerve opioid receptors (to reduce pain) \\
\hline TENS unit & Transcutaneous electrical nerve stimulus (to block pain afferents (gate theory)) \\
\hline Spinal stimulator & Spinal cord stimulation $\left(C_{7} / T_{1}, T_{1} / T_{2}\right.$; to block pain afferents (gate theory)) \\
\hline
\end{tabular}

is personalised for individual patients based on the underlying pathophysiological mechanisms responsible for INOCA (figs 1 and 5). Thus, specific pathophysiologically based treatment approaches are detailed below.

\section{Establish the responsible pathophysiological mechanisms}

Is it INOCA?

Before initiating treatment, doctors should be confident of the clinical diagnosis, so that noncardiac and non-ischaemic causes of the chest pain can be excluded (figs 3 and 5). However, even in patients with an established coronary vasomotor disorder, discerning clinicians should continue to monitor for alternative causes, especially if chest pain characteristics have changed (eg, new gastrooesophageal reflux). Moreover, patients can develop obstructive coronary artery disease over time, so this possibility should be reconsidered.

Is it macrovascular or microvascular dysfunction? Although some INOCA treatments are effective in both coronary macrovascular and microvascular dysfunction, others are more specific to one of these mechanisms. Hence treatments should be prescribed on the basis of clinical evidence, which targets one of these mechanisms or disease entities (table 3 and fig 5). As described above, functional coronary angiography would be a good diagnostic tool for evaluating the responsible pathophysiological mechanisms in patients with INOCA but is not often performed despite guideline recommendations. ${ }^{108}$ Moreover, functional coronary angiography could reveal that a patient has both macrovascular and microvascular dysfunction.

In the absence of functional coronary angiography, classic clinical presentations might assist doctors in distinguishing vasospastic angina from coronary microvascular disorders; however, these traditional methods have not undergone rigorous validation. The classic features of vasospastic angina are rest angina with ST elevation, both rapidly resolving with sublingual nitrates. Nocturnal angina and an association with migraines havealso been reported. ${ }^{109}$ In contrast, syndrome $\mathrm{X}$ is classically described as pain occurring on effort, which has a variable response to sublingual nitrates and frequently occurs 


\section{Table 3 | Vasospastic angina studies}

\begin{tabular}{|c|c|c|c|c|c|c|}
\hline Study & No & Intervention & Inclusion criteria & $\begin{array}{l}\text { Coronary } \\
\text { microvascular } \\
\text { dysfunction }\end{array}$ & Study design & Impact on outcomes \\
\hline \multicolumn{7}{|c|}{ Calcium channel blockers } \\
\hline Heupler $(1979)^{45}$ & 8 & Nifedipine & $\begin{array}{l}\text { Rest angina, NOCA, } \\
\text { transient STE }\end{array}$ & Bedside test & $\begin{array}{l}\text { Observational, non- } \\
\text { randomised }\end{array}$ & $\begin{array}{l}\text { Nifedipine reduced mean monthly angina episodes } \\
\text { from } 104 \text { to eight }\end{array}$ \\
\hline $\begin{array}{l}\text { Ascherman } \\
(1990)^{46}\end{array}$ & 17 & Nifedipine & $\begin{array}{l}\text { Angina, NOCA, } \\
\text { transient STE }\end{array}$ & $\begin{array}{l}\text { Spontaneous spasm on } \\
\text { angiography }\end{array}$ & $\begin{array}{l}\text { Double blind, } \\
\text { comparative crossover } \\
\text { RCT }\end{array}$ & $\begin{array}{l}\text { Nifedipine reduced mean weekly angina episodes from } \\
43 \text { to eight, after six weeks }(P<0.001)\end{array}$ \\
\hline Chahine $(1993)^{47}$ & 52 & Amlodipine & $\begin{array}{l}\text { Rest angina, transient } \\
\text { STE }\end{array}$ & $\begin{array}{l}\text { Provoked spasm on } \\
\text { angiography or beside }\end{array}$ & $\begin{array}{l}\text { Double blind, placebo } \\
\text { controlled, crossover } \\
\text { RCT }\end{array}$ & $\begin{array}{l}\text { Amlodipine reduced mean daily angina episodes by } \\
55 \%(\text { placebo }=15 \%) \text { at four weeks }(P=0.009)\end{array}$ \\
\hline Higuma $(2010)^{48}$ & 37 & $\begin{array}{l}\text { Nifedipine CR; } \\
\text { diltiazem R }\end{array}$ & $\begin{array}{l}\text { Rest angina, NOCA, } \\
\text { ischaemic ECG }\end{array}$ & $\begin{array}{l}\text { Provoked spasm on } \\
\text { angiography }\end{array}$ & $\begin{array}{l}\text { Open label, } \\
\text { comparative RCT }\end{array}$ & $\begin{array}{l}\text { Mean weekly angina episodes reduced over } 12 \text { weeks } \\
\text { for nifedipine }(2.6 \text { to } 0.4, P<0.05) \text { and diltiazem }(2.7 \\
\text { to } 0.3, P<0.05)\end{array}$ \\
\hline Oikawa $(2010)^{49}$ & 30 & $\begin{array}{l}\text { Nifedipine; } \\
\text { benidpine }\end{array}$ & $\begin{array}{l}\text { Rest angina, NOCA, } \\
\text { ischaemic ECG }\end{array}$ & $\begin{array}{l}\text { Provoked spasm on } \\
\text { angiography }\end{array}$ & Comparative RCT & $\begin{array}{l}\text { Nifedipine reduced mean weekly angina episodes } \\
\text { from } 3.5(S D \pm 7.7) \text { to } 0.1( \pm 0.4) \text {, at } 8 \text { weeks }(P<0.001) \text {; } \\
\text { benidipine did not improve angina episodes ( } 1.4 \\
( \pm 1.1) \text { to } 0.6( \pm 0.8), P>0.5)\end{array}$ \\
\hline Kook $(2020)^{50}$ & 48 & $\begin{array}{l}\text { Diltiazem; } \\
\text { nebivolol }\end{array}$ & Rest angina, NOCA & $\begin{array}{l}\text { Provoked spasm on } \\
\text { angiography }\end{array}$ & $\begin{array}{l}\text { Double blind, parallel } \\
\text { group RCT }\end{array}$ & $\begin{array}{l}\text { Change (\%) in diameter from baseline acetylcholine } \\
\text { provocation test. Suppression of acetylcholine } \\
\text { constriction: diltiazem }(68 \pm 13 \%) \text {, nebivolol ( } 50 \\
\pm 8 \% \text { ), diltiazem + nebivolol }(47 \pm 12 \% \text { ) at } 12 \text { weeks } \\
(\mathrm{P}=0.054) \text {; SAQ scores improved in overall study } \\
\text { population ( } 80.3 \pm 7.9 \text { to } 85.4 \pm 7.8, P<0.001 \text { ); no } \\
\text { significant difference in SAQ between diltiazem and } \\
\text { nebivolol }\end{array}$ \\
\hline
\end{tabular}

\section{$\beta$ adrenergic agents}

Shimizu $(1993)^{51} 10$

Long acting nitrates

$(1990)^{46}$

17 Isosorbide

dinitrate
Rest angina, NOCA, ischaemic ECG

\section{Provoked spasm on} angiography

\section{Placebo controlled RCT}

Denopamine reduced mean daily angina frequency compared with placebo $(0.6(\mathrm{SD} \pm 1.2)$ v $2.2( \pm 1.3)$ P<0.005)

\section{Angina, NOCA,}

transient STE

Double blind,

Isosorbide dinitrate reduced mean weekly angina episodes from 43 to four, after six weeks (P<0.001)
Ascherman
Spontaneous spasm on angiography RCT

\begin{tabular}{llllll}
\hline Kishida $(1987)^{41}$ & 32 & Nicorandil & $\begin{array}{l}\text { Angina, NOCA, } \\
\text { transient STE }\end{array}$ & NA & $\begin{array}{l}\text { Single blind, placebo } \\
\text { controlled, crossover } \\
\text { RCT }\end{array}$ \\
\hline $\begin{array}{l}\text { Lablanche } \\
(1992)^{42}\end{array}$ & 13 & $\begin{array}{l}\text { Nicorandil; } \\
\text { nifedipine }\end{array}$ & $\begin{array}{l}\text { Angina, NOCA, } \\
\text { transient STE }\end{array}$ & $\begin{array}{l}\text { Spontaneous or } \\
\text { provoked spasm on } \\
\text { angiography }\end{array}$ & $\begin{array}{l}\text { Single blind, placebo } \\
\text { controlled, crossover } \\
\text { RCT }\end{array}$
\end{tabular}

\section{Statins}

\begin{tabular}{llllll}
\hline Yasue $(2008)^{52}$ & 64 & Fluvastatin & $\begin{array}{l}\text { Angina, NOCA, } \\
\text { positive stress test, } \\
\text { background CCB } \\
\text { treatment }\end{array}$ & $\begin{array}{l}\text { Acetylcholine induced } \\
\text { spasm on angiography }\end{array}$ & Open label RCT \\
\hline Kim $(2019)^{53}$ & 100 & $\begin{array}{l}\text { Atorvastatin; } \\
\text { sarpogrelate }\end{array}$ & $\begin{array}{l}\text { Chest pain, NOCA, } \\
\text { substantial ST-T wave } \\
\text { change }\end{array}$ & $\begin{array}{l}\text { Provoked spasm on } \\
\text { angiography }\end{array}$ & $\begin{array}{l}\text { Placebo controlled RCT } \\
\text { with } 2 \times 2 \text { design }\end{array}$ \\
\hline
\end{tabular}

Oestrogens

Kawano $(2001)^{54} 15 \quad 17-\beta$ oestradiol $\quad$ Rest angina, NCA, Provoked spasm on $\quad$ Placebo controlled RCT
transient ST elevation angiography

\section{Potassium channel openers} Nicorandil reduced mean daily angina episodes compared to placebo (placebo=mean $3.6(\mathrm{SE} \pm 0.4)$ per day, nicorandil=0.7 $( \pm 0.2)$ per day, P<0.001) Nicorandil suppressed inducible spasm in $92 \%$ of patients (all inducible on placebo); nifedipine suppressed inducible spasm in $69 \%$ of patients (all inducible on placebo)

$75 \%$ of patients in the fluvastatin group became asymptomatic after 6 months ( $\mathrm{P}<0.001)$, compared to $70 \%$ of control patients ( $p<0.001$ )

Atorvastatin and sarpogrelate did not affect readmission rate compared with placebo (sarpogrelate + atorvastatin $47 \%$, sarpogrelate $58 \%$, atorvastatin $40 \%$ and placebo $56 \%, P=0.74)$

$17-\beta$ oestradiol suppressed hyperventilation induced angina in all patients on the third day of supplementation

\begin{tabular}{|c|c|c|c|c|c|c|}
\hline \multicolumn{7}{|c|}{ Exercise therapy or lifestyle } \\
\hline $\begin{array}{l}\text { Morikawa } \\
(2013)^{55}\end{array}$ & 26 & Exercise training & $\begin{array}{l}\text { Rest angina, NOCA, } \\
\text { ischaemic ECG }\end{array}$ & $\begin{array}{l}\text { Provoked spasm on } \\
\text { angiography }\end{array}$ & $\begin{array}{l}\text { Non-randomised } \\
\text { intervention }\end{array}$ & $\begin{array}{l}\text { Exercise training improved median angina episodes } \\
\text { from } 2(\text { IQR } 1-7) \text { to } 0(0-2) \text { at five days ( } P<0.001)\end{array}$ \\
\hline \multicolumn{7}{|l|}{ Novel agents } \\
\hline Huckell $(1981)^{43}$ & 18 & Perhexiline & Rest angina, STE & $\begin{array}{l}\text { Transient ST elevation, } \\
\text { spasm on angiography } \\
\text { in } 13\end{array}$ & $\begin{array}{l}\text { Non-randomised } \\
\text { interventions }\end{array}$ & Perhexiline reduced angina episodes \\
\hline Morita $(2014)^{56}$ & 73 & Pioglitazone & $\begin{array}{l}\text { Rest angina, NOCA, } \\
\text { ischaemic ECG, } \\
\text { background CCB } \\
\text { treatment }\end{array}$ & $\begin{array}{l}\text { Acetylcholine induced } \\
\text { spasm on angiography }\end{array}$ & Open label RCT & $\begin{array}{l}\text { Pioglitazone improved angina episodes by } 75 \% \text { but } \\
\text { was not different compared to placebo }(74 \%, \text { P } 0.001) \\
\text { at six months }\end{array}$ \\
\hline Shin $(2014)^{57}$ & 50 & Cilostazol & $\begin{array}{l}\text { Chest pain, NOCA, } \\
\text { ischaemic ECG, } \\
\text { background CCB } \\
\text { treatment }\end{array}$ & $\begin{array}{l}\text { Provoked spasm on } \\
\text { angiography }\end{array}$ & $\begin{array}{l}\text { Double blind, placebo } \\
\text { controlled, RCT }\end{array}$ & $\begin{array}{l}\text { Cilostazol reduced weekly angina episodes (mean } 66 \\
\mathrm{SD} \pm 87 \%) \text { compared to placebo }(18 \pm 140 \%, P=0.009) \\
\text { at six months }\end{array}$ \\
\hline
\end{tabular}

NOCA=non-obstructive coronary arteries; $N C A=$ normal coronary arteries; $S T E=S T$ segment elevation; $R C T=$ randomised clinical trial; $C F R=$ coronary flow reserve; $C S F P=$ coronary slow flow phenomenon; $\mathrm{SAQ}=$ Seattle Angina Questionnaire; $C R=$ cardiac rehabilitation; $R=$ sustained release; $E C G=$ electrocardiogram; $C C B=c a l c i u m$ channel blockers; $N A=n o t$ available; $S D=s t a n d a r d$ deviation; $\mathrm{SE}=$ standard error; $\mathrm{IQR}=$ interquartile range. 


\section{Table 4 | Coronary microvascular disorder studies}

\begin{tabular}{|c|c|c|c|c|c|c|}
\hline & No & Intervention & Inclusion criteria & $\begin{array}{l}\text { Coronary } \\
\text { microvascular } \\
\text { dysfunction }\end{array}$ & Study design & Impact on outcomes \\
\hline \multicolumn{7}{|c|}{ Calcium channel blockers } \\
\hline Cannon $(1985)^{58}$ & 26 & $\begin{array}{l}\text { Verapamil; } \\
\text { nifedipine }\end{array}$ & Chest pain, NCA & Abnormal CFR & $\begin{array}{l}\text { Double blind, placebo } \\
\text { controlled, crossover } \\
\text { RCT }\end{array}$ & $\begin{array}{l}\text { CCBs improved angina episodes compared to placebo (mean } \\
21 \mathrm{SD} \pm 21 \vee 35 \pm 27, \mathrm{P}<0.001 \text { ) }\end{array}$ \\
\hline Cannon $(1990)^{59}$ & 11 & Lidoflazine & Chest pain, NCA & Abnormal CFR & $\begin{array}{l}\text { Double blind, placebo } \\
\text { controlled, RCT }\end{array}$ & $\begin{array}{l}\text { Lidoflazine did not affect angina episodes (mean }-9 \mathrm{SD} \pm 37 \text {, } \\
\text { P>0.05) at seven weeks }\end{array}$ \\
\hline Beltrame $(2004)^{60}$ & 20 & Mibefradil & Angina, NOCA & CSFP & $\begin{array}{l}\text { Double blind, placebo } \\
\text { controlled, crossover } \\
\text { RCT }\end{array}$ & $\begin{array}{l}\text { Mibefradil reduced monthly angina episodes by } 56 \% \text { compared } \\
\text { to placebo }(P<0.001)\end{array}$ \\
\hline Zhang $(2014)^{61}$ & 66 & $\begin{array}{l}\text { Diltiazem; } \\
\text { fluvastatin }\end{array}$ & $\begin{array}{l}\text { Angina, NCA, } \\
\text { positive stress test }\end{array}$ & Not required & Randomised trial & $\begin{array}{l}\text { Diltiazem + fluvastatin improved mean exercise time at } 90 \text { days } \\
\text { compared to either drug alone (mean } 446 \mathrm{SD} \pm 164 \vee 250 \pm 104 \\
\text { seconds, } P<0.05 \text { ) }\end{array}$ \\
\hline \multicolumn{7}{|c|}{ Beta adrenergic agents } \\
\hline Lanza $(1999)^{35}$ & 10 & $\begin{array}{l}\text { Atenolol; } \\
\text { amlodipine; } \\
\text { isosorbide } \\
\text { mononitrate }\end{array}$ & $\begin{array}{l}\text { Exercise angina, } \\
\text { NCA, positive stress } \\
\text { test }\end{array}$ & Not assessed & $\begin{array}{l}\text { Double blind, } \\
\text { placebo controlled, } \\
\text { comparative RCT }\end{array}$ & $\begin{array}{l}\text { Atenolol reduced monthly angina episodes from mean } 24 \\
(\mathrm{SD} \pm 18) \text { to } 15( \pm 13) ; \mathrm{P}<0.05) \text {; amlodipine and isosorbide } \\
\text { mononitrate had no impact on angina episodes }\end{array}$ \\
\hline Sen $(2009)^{62}$ & 38 & $\begin{array}{l}\text { Nebivolol; } \\
\text { metoprolol }\end{array}$ & $\begin{array}{l}\text { Angina, NCA, } \\
\text { transient ST } \\
\text { depression during } \\
\text { exercise }\end{array}$ & Not required & Single blind RCT & $\begin{array}{l}\text { Nebivolol improved CCS angina class in } 73 \% \text { patients and total } \\
\text { exercise time; metoprolol improved CCS angina class in } 47 \% \\
\text { patients but did not affect exercise }\end{array}$ \\
\hline \multicolumn{7}{|l|}{ Long acting nitrates } \\
\hline Bugiardini $(1993)^{63}$ & 16 & $\begin{array}{l}\text { Isosorbide } \\
\text { dinitrate }\end{array}$ & $\begin{array}{l}\text { Angina, NCA, } \\
\text { positive stress test }\end{array}$ & Not required & $\begin{array}{l}\text { Non-randomised } \\
\text { intervention }\end{array}$ & $\begin{array}{l}\text { Isosorbide dinitrate decreased time to angina on exercise } \\
\text { testing compared to baseline (mean } 477 \mathrm{SD} \pm 93 \vee 561 \pm 108 \\
\text { seconds, } P<0.002 \text { ) }\end{array}$ \\
\hline Russo $(2013)^{64}$ & 29 & $\begin{array}{l}\text { Isosorbide } \\
\text { dinitrate }\end{array}$ & $\begin{array}{l}\text { Angina, NCA, } \\
\text { positive stress test }\end{array}$ & Not required & $\begin{array}{l}\text { Non-randomised } \\
\text { intervention }\end{array}$ & Isosoribide dintrate did not affect exercise duration \\
\hline \multicolumn{7}{|c|}{ Potassium channel openers } \\
\hline Yamabe $(1995)^{65}$ & 11 & Nicorandil & $\begin{array}{l}\text { Angina, NCA, } \\
\text { positive stress test }\end{array}$ & Not required & $\begin{array}{l}\text { Non-randomised } \\
\text { intervention }\end{array}$ & $\begin{array}{l}\text { Nicorandil improved the extent of ischaemia score on } \\
{ }^{201} \text { Thallium scintigraphy (before, mean } 0.37 \mathrm{SD} \pm 0.22 \text {; after, } \\
0.20 \pm 0.15 ; \mathrm{P}<0.05 \text { ) }\end{array}$ \\
\hline Chen $(1997)^{40}$ & 13 & Nicorandil & $\begin{array}{l}\text { Angina, NCA, } \\
\text { positive stress test }\end{array}$ & Abnormal CFR & $\begin{array}{l}\text { Double blind, placebo } \\
\text { controlled, crossover } \\
\text { RCT }\end{array}$ & $\begin{array}{l}\text { Nicorandil improved total exercise time (mean } 443 \mathrm{SD} \pm 78 \mathrm{v} \\
405 \pm 64 \text { seconds, } \mathrm{P}=0.036) \text { and time to } 1 \mathrm{~mm} \text { ST depression } \\
(342 \pm 104 \mathrm{v} 273 \pm 72 \text { seconds, } \mathrm{P}=0.026) \text { compared with } \\
\text { placebo }\end{array}$ \\
\hline \multicolumn{7}{|l|}{ Statins } \\
\hline $\begin{array}{l}\text { Kayikcioglu } \\
(2003)^{66}\end{array}$ & 40 & Pravastatin & $\begin{array}{l}\text { Angina, NCA, } \\
\text { positive stress test }\end{array}$ & Not required & $\begin{array}{l}\text { Single blind, placebo } \\
\text { controlled RCT }\end{array}$ & $\begin{array}{l}\text { Pravastatin improved mean exercise time (mean } 530 \mathrm{SD} \pm 162 \\
\vee 585 \pm 165 \text { seconds, } \mathrm{P}<0.05) \text { and time to } 1 \mathrm{~mm} \text { ST depression } \\
(267 \pm 105 \vee 419 \pm 162 \text { seconds, } P<0.05) \text { at } 90 \text { days }\end{array}$ \\
\hline Fabian $(2004)^{67}$ & 40 & Simvastatin & $\begin{array}{l}\text { Angina, NCA, } \\
\text { positive stress test }\end{array}$ & Not required & $\begin{array}{l}\text { Open label, placebo } \\
\text { controlled RCT }\end{array}$ & $\begin{array}{l}\text { Simvastatin improved time to } 1 \mathrm{~mm} \text { ST depression (mean } 4.45 \\
\mathrm{SD} \pm 0.39 \text { v } 5.33 \pm 0.27 \text { minutes, } \mathrm{P}<0.001 \text { ) at } 12 \text { weeks, but was } \\
\text { unchanged with placebo }\end{array}$ \\
\hline \multicolumn{7}{|l|}{ Oestrogen } \\
\hline $\begin{array}{l}\text { Bairey Merz } \\
(2010)^{68}\end{array}$ & $\begin{array}{l}35 \\
\text { WO }\end{array}$ & $\begin{array}{l}\text { Norethindrone } \\
\text { or ethinyl } \\
\text { estradiol }\end{array}$ & $\begin{array}{l}\text { Angina, NOCA, } \\
\text { positive stress test }\end{array}$ & Abnormal CFR & $\begin{array}{l}\text { Double blind, placebo } \\
\text { controlled RCT }\end{array}$ & $\begin{array}{l}\text { Oestrogen treatment increased angina-free status compared to } \\
\text { placebo }(41 \% \vee 22 \% \text { angina free, } \mathrm{P}=0.02) \text { at } 12 \text { weeks }\end{array}$ \\
\hline \multicolumn{7}{|c|}{ Renin/angiotensin/aldosterone inhibitors } \\
\hline Kaski $(1994)^{69}$ & 19 & Enalapril & $\begin{array}{l}\text { Angina, NCA, } \\
\text { positive stress test }\end{array}$ & Abnormal CFR & $\begin{array}{l}\text { Single blind, placebo } \\
\text { controlled, crossover } \\
\text { RCT }\end{array}$ & $\begin{array}{l}\text { Enalapril improved total exercise time (mean } 779 \mathrm{SD} \pm 141 \mathrm{~V} \\
690 \pm 148 \text { seconds, } \mathrm{P}=0.006 \text { ) at two weeks }\end{array}$ \\
\hline Motz $(1996)^{70}$ & 15 & Enalapril & $\begin{array}{l}\text { NCA, abnormal } \\
\text { resting ECG or } \\
\text { positive stress test }\end{array}$ & Not required & $\begin{array}{l}\text { Non-randomised } \\
\text { intervention }\end{array}$ & $\begin{array}{l}\text { Enalapril improved CCS angina class (mean } 2.5 \mathrm{SD} \pm 0.6 \text { to } 1.5 \\
\pm 0.6, \mathrm{P}<0.01 \text { ) at } 12 \text { months }\end{array}$ \\
\hline Chen $(2002)^{71}$ & 20 & Enalapril & $\begin{array}{l}\text { Angina, NCA, } \\
\text { positive stress test }\end{array}$ & Not required & $\begin{array}{l}\text { Double blind, placebo } \\
\text { controlled RCT }\end{array}$ & $\begin{array}{l}\text { Enalapril reduced weekly angina episodes (mean } 1.6 \mathrm{SD} \pm 0.5 \mathrm{v} \\
0.5 \pm 0.4, \mathrm{P}<0.05 \text { ) at eight weeks }\end{array}$ \\
\hline Pauly $(2011)^{72}$ & 78 & Quinapril & Angina, NOCA & Abnormal CFR & $\begin{array}{l}\text { Double blind, placebo } \\
\text { controlled RCT }\end{array}$ & $\begin{array}{l}\text { Quinapril reduced angina episodes at } 16 \text { weeks compared to } \\
\text { placebo }(P=0.037)\end{array}$ \\
\hline Bavry $(2014)^{73}$ & $\begin{array}{l}41 \\
\text { WO }\end{array}$ & Eplerenone & $\begin{array}{l}\text { Angina, NCA, } \\
\text { positive stress test }\end{array}$ & Abnormal CFR & $\begin{array}{l}\text { Double blind, placebo } \\
\text { controlled RCT }\end{array}$ & $\begin{array}{l}\text { Eplerenone did not affect angina episodes at } 16 \text { weeks, } \\
\text { compared with placebo }\end{array}$ \\
\hline Michelsen $(2018)^{74}$ & $\begin{array}{l}63 \\
\text { WO }\end{array}$ & Ramipril & $\begin{array}{l}\text { Angina, NCA, not } \\
\text { required }\end{array}$ & Abnormal CFR & $\begin{array}{l}\text { Double blind, placebo } \\
\text { controlled RCT }\end{array}$ & $\begin{array}{l}\text { Ramapril did not affect angina episodes at } 24 \text { weeks, compared } \\
\text { with placebo }\end{array}$ \\
\hline \multicolumn{7}{|c|}{ Nitric oxide modulators } \\
\hline Lerman $(1998)^{75}$ & 26 & $\begin{array}{l}\text { L-arginine } \\
\text { infusion }\end{array}$ & Chest pain, NOCA & Not assessed & $\begin{array}{l}\text { Double blind, placebo } \\
\text { controlled RCT }\end{array}$ & $\begin{array}{l}\text { L-arginine improved qualitative symptom scores compared with } \\
\text { placebo }(P<0.05)\end{array}$ \\
\hline Denardo $(2011)^{76}$ & 23 & Sildenafil & Chest pain, NOCA & CFR $\leq 3.0$ & $\begin{array}{l}\text { Non-randomised } \\
\text { intervention }\end{array}$ & Sildenafil improved CFR in patients with baseline CFR $<2.5$ \\
\hline
\end{tabular}




\section{Table 4 | Continued}

\begin{tabular}{|c|c|c|c|c|c|c|}
\hline & No & Intervention & Inclusion criteria & $\begin{array}{l}\text { Coronary } \\
\text { microvascular } \\
\text { dysfunction }\end{array}$ & Study design & Impact on outcomes \\
\hline \multicolumn{7}{|l|}{ a adrenergic agents } \\
\hline Cannon $(1994)^{77}$ & 60 & $\begin{array}{l}\text { Clonidine; } \\
\text { imipramine }\end{array}$ & $\begin{array}{l}\text { Refractory chest } \\
\text { pain, NCA }\end{array}$ & Not required & $\begin{array}{l}\text { Double blind, placebo } \\
\text { controlled RCT }\end{array}$ & $\begin{array}{l}\text { Imipramine reduced mean angina episodes by mean } 52 \\
(\mathrm{SD} \pm 25 \%) \text { at three weeks }(\mathrm{P}=0.03) \text {; clonidine had no impact on } \\
\text { angina episodes }\end{array}$ \\
\hline Bøtker $(1998)^{78}$ & 16 & Doxazosin & $\begin{array}{l}\text { Angina, NCA, } \\
\text { positive stress test }\end{array}$ & Not assessed & $\begin{array}{l}\text { Double blind, placebo } \\
\text { controlled, crossover } \\
\text { RCT }\end{array}$ & Doxazosin did not affect exercise induced angina at 10 weeks \\
\hline Rosen $(1999)^{79}$ & 15 & Doxazosin & $\begin{array}{l}\text { Angina, NCA, } \\
\text { positive stress test }\end{array}$ & Not required & $\begin{array}{l}\text { Double blind, placebo } \\
\text { controlled RCT }\end{array}$ & $\begin{array}{l}\text { Doxazosin did not affect total exercise time or time to } 1 \mathrm{~mm} \mathrm{ST} \\
\text { depression compared to placebo, at seven days }\end{array}$ \\
\hline \multicolumn{7}{|c|}{ Novel anti-anginal agents } \\
\hline Topal $(2006)^{80}$ & 48 & Trimetazidine & $\begin{array}{l}\text { Ischaemic } \\
\text { symptoms, NCA }\end{array}$ & CSFP & $\begin{array}{l}\text { Double blind, placebo } \\
\text { controlled RCT }\end{array}$ & $\begin{array}{l}\text { Trimetazidine improved exercise time to angina (before, mean } \\
339 \mathrm{SD} \pm 73 \mathrm{v} \text { after, } 421 \pm 71 \text { seconds; } P=0.02 \text { ) and the number } \\
\text { of angina-free patients at four weeks, compared to placebo } \\
(P=0.03)\end{array}$ \\
\hline Mehta $(2011)^{38}$ & $\begin{array}{l}20 \\
\text { WO }\end{array}$ & Ranolazine & $\begin{array}{l}\text { Angina, NOCA, } \\
\text { positive stress test }\end{array}$ & Abnormal CFR & $\begin{array}{l}\text { Double blind, placebo } \\
\text { controlled, crossover } \\
\text { RCT }\end{array}$ & $\begin{array}{l}\text { Ranolazine improved angina related physical limitation } \\
\text { compared to placebo (SAQ physical limitation scores, median } \\
92 \text { (range 79-98) v } 83(67-97) ; P=0.046) \text { but not angina } \\
\text { frequency }\end{array}$ \\
\hline Villano $(2013)^{39}$ & 45 & $\begin{array}{l}\text { Ranolazine; } \\
\text { ivabradine }\end{array}$ & $\begin{array}{l}\text { Exercise angina, } \\
\text { NOCA, positive } \\
\text { stress test }\end{array}$ & Abnormal CFR & $\begin{array}{l}\text { Double blind, placebo } \\
\text { controlled RCT }\end{array}$ & $\begin{array}{l}\text { Ranolazine improved SAQ angina frequency at four weeks } \\
\text { (mean } 61 \mathrm{SD} \pm 12 \vee 81 \pm 17) \text { to a greater extent than ivabradine } \\
(64 \pm 14 \vee 73 \pm 18) \text { or placebo }(73 \pm 17 \vee 71 \pm 18 \text {; } \mathrm{P}<0.001)\end{array}$ \\
\hline $\begin{array}{l}\text { Bairey Merz } \\
(2016)^{81}\end{array}$ & $\begin{array}{l}128 \\
\text { WO }\end{array}$ & Ranolazine & $\begin{array}{l}\text { Suspected } \\
\text { ischaemia, NOCA, } \\
\text { positive stress test }\end{array}$ & Abnormal CFR & $\begin{array}{l}\text { Double blind, placebo } \\
\text { controlled, crossover } \\
\text { RCT }\end{array}$ & $\begin{array}{l}\text { Ranolazine SAQ angina frequency at two weeks was not } \\
\text { different to placebo (mean difference }-0.08 \text { ( } 95 \% \text { confidence } \\
\text { interval }-4.18,4.34), P=0.97)\end{array}$ \\
\hline Ahmed $(2017)^{82}$ & 7 & Ranolazine & $\begin{array}{l}\text { Angina, NOCA, } \\
\text { positive stress test }\end{array}$ & $\begin{array}{l}\text { Abnormal index } \\
\text { of microvascular } \\
\text { resistance }\end{array}$ & $\begin{array}{l}\text { Non-randomised } \\
\text { intervention }\end{array}$ & $\begin{array}{l}\text { Ranolazine improved SAQ angina frequency scores at four } \\
\text { weeks compared to baseline }(P=0.04)\end{array}$ \\
\hline Rambarat (2019) ${ }^{83}$ & 81 & Ranolazine & $\begin{array}{l}\text { Angina, NOCA, } \\
\text { positive stress test }\end{array}$ & Abnormal CFR & $\begin{array}{l}\text { Double blind, placebo } \\
\text { controlled, crossover } \\
\text { RCT }\end{array}$ & $\begin{array}{l}\text { Ranolazine improved SAQ angina frequency compared to } \\
\text { placebo (mean difference } 5.76 \mathrm{SD} \pm 11.51 \mathrm{~V}-0.86 \pm 13.54 \text {, } \\
\text { confidence interval } 0.28 \text { to } 12.96, \mathrm{P}=0.007 \text { ) at two weeks, in } \\
\text { patients with low CFR }\end{array}$ \\
\hline $\operatorname{Lim}(2017)^{84}$ & 19 & Allopurinol & $\begin{array}{l}\text { Angina, NCA, } \\
\text { positive stress test }\end{array}$ & Not required & $\begin{array}{l}\text { Double blind, placebo } \\
\text { controlled, crossover } \\
\text { RCT }\end{array}$ & $\begin{array}{l}\text { Allopurinol did not affect total exercise time, compared with } \\
\text { placebo }\end{array}$ \\
\hline
\end{tabular}

Exercise therapy or lifestyle factors

\begin{tabular}{|c|c|c|c|c|c|c|}
\hline Eriksson $(2000)^{85}$ & 26 & Exercise training & $\begin{array}{l}\text { Angina, NCA, } \\
\text { positive stress test }\end{array}$ & Not required & $\begin{array}{l}\text { Parallel design (usual } \\
\text { care) RCT }\end{array}$ & $\begin{array}{l}\text { Exercise training improved time to angina relative to baseline } \\
\text { (mean } 4 \mathrm{SD} \pm 1 \vee 6 \pm 1 \text { minute, } \mathrm{P}=0.01 \text { ) }\end{array}$ \\
\hline $\begin{array}{l}\text { Tyni-Lenne } \\
(2002)^{86}\end{array}$ & $\begin{array}{l}24 \\
\text { WO }\end{array}$ & Exercise training & $\begin{array}{l}\text { Angina, NCA, } \\
\text { positive stress test }\end{array}$ & Not required & $\begin{array}{l}\text { Parallel design (usual } \\
\text { care) RCT }\end{array}$ & $\begin{array}{l}\text { Exercise training improved walk distance relative to baseline } \\
\text { (mean } 555 \mathrm{SD} \pm 47 \vee 587 \pm 49 \text { metres, } \mathrm{P}<0.006)\end{array}$ \\
\hline Asbury $(2008)^{87}$ & $\begin{array}{l}64 \\
\text { WO }\end{array}$ & $\begin{array}{l}\text { Cardiac } \\
\text { rehabilitation }\end{array}$ & $\begin{array}{l}\text { Chest pain, NCA, } \\
\text { positive stress test }\end{array}$ & Not required & $\begin{array}{l}\text { Parallel design (usual } \\
\text { care) RCT }\end{array}$ & $\begin{array}{l}\text { Cardiac rehabilitation improved symptom severity at eight } \\
\text { weeks (before, mean } 2.0 \mathrm{SD} \pm 0.8 v \text { after } 1.26 \pm 1.1, \mathrm{P}=0.009 \text { ) }\end{array}$ \\
\hline Fiezi $(2012)^{88}$ & 40 & $\begin{array}{l}\text { Cardiac } \\
\text { rehabilitation }\end{array}$ & $\begin{array}{l}\text { Angina, NCA, } \\
\text { positive stress test }\end{array}$ & Not required & $\begin{array}{l}\text { Parallel design (usual } \\
\text { care) RCT }\end{array}$ & $\begin{array}{l}\text { Cardiac rehabilitation improved physical functioning scores } \\
\text { compared to controls (mean } 59 \mathrm{SD} \pm 8 \vee 36 \pm 13, \mathrm{P}<0.05 \text { ) }\end{array}$ \\
\hline Fiezi $(2012)^{88}$ & 40 & $\begin{array}{l}\text { Relaxation } \\
\text { therapy }\end{array}$ & $\begin{array}{l}\text { Angina, NCA, } \\
\text { positive stress test }\end{array}$ & Not required & $\begin{array}{l}\text { Parallel design (usual } \\
\text { care) RCT }\end{array}$ & $\begin{array}{l}\text { Relaxation therapy improved physical functioning scores } \\
\text { compared to controls (mean } 51 \mathrm{SD} \pm 13 \vee 30 \pm 9, \mathrm{P}<0.05 \text { ) }\end{array}$ \\
\hline $\begin{array}{l}\text { de Carvalho } \\
(2015)^{89}\end{array}$ & 12 & Exercise training & $\begin{array}{l}\text { Angina, NCA, } \\
\text { positive stress test }\end{array}$ & Not required & $\begin{array}{l}\text { Non-randomised } \\
\text { intervention }\end{array}$ & $\begin{array}{l}\text { Exercise training improved physical functioning scores relative } \\
\text { to baseline (mean } 88 \mathrm{SD} \pm 9 \text { v } 45 \pm 26, P<0.001) \text {. }\end{array}$ \\
\hline Szot $(2015)^{90}$ & $\begin{array}{l}55 \\
\text { WO }\end{array}$ & $\begin{array}{l}\text { Cardiac } \\
\text { rehabilitation }\end{array}$ & $\begin{array}{l}\text { Angina, NOCA, } \\
\text { positive stress test }\end{array}$ & Not required & $\begin{array}{l}\text { Non-randomised } \\
\text { intervention }\end{array}$ & $\begin{array}{l}\text { Cardiac rehabilitation improved exercise duration of exercise } \\
\text { relative to baseline (mean } 760 S D \pm 142 \text { v } 636 \pm 157 \text { seconds, } \\
P<0.001 \text { ) }\end{array}$ \\
\hline Bove $(2020)^{91}$ & $\begin{array}{l}56 \\
\text { WO }\end{array}$ & $\begin{array}{l}\text { Exercise and risk } \\
\text { factor control }\end{array}$ & Angina, NOCA & Abnormal CFR & $\begin{array}{l}\text { Parallel design (usual } \\
\text { care) RCT }\end{array}$ & $\begin{array}{l}\text { Lifestyle risk factor control improved SAQ angina frequency } \\
\text { scores compared to usual care (difference } 12.91 \text { ( } 95 \% \mathrm{Cl} 4.91 \\
\text { to } 20.90), \mathrm{P}=0.002 \text { ) }\end{array}$ \\
\hline \multicolumn{7}{|l|}{ Devices } \\
\hline Eliasson $(1993)^{92}$ & 12 & $\begin{array}{l}\text { Spinal cord } \\
\text { stimulation }\end{array}$ & $\begin{array}{l}\text { Angina, NCA, } \\
\text { positive stress test }\end{array}$ & Not assessed & $\begin{array}{l}\text { Non-randomised } \\
\text { intervention }\end{array}$ & $\begin{array}{l}\text { Spinal cord stimulation improves exercise test time to angina } \\
\text { (before } 2.7 \pm 1.9 \text {, after } 5.4 \pm 2.2 \text { minutes, } P<0.01 \text { ) and time to } \\
1 \mathrm{~mm} \text { ST depression (before } 2.4 \pm 1.6 \text {, after } 3.5 \pm 1.9 \text { minutes, } \\
P<0.01 \text { ) }\end{array}$ \\
\hline Jessurun $(2003)^{93}$ & 8 & TENS & $\begin{array}{l}\text { Angina, NCA, } \\
\text { positive stress test }\end{array}$ & Not assessed & $\begin{array}{l}\text { Non-randomised } \\
\text { intervention }\end{array}$ & $\begin{array}{l}\text { TENS reduced angina episodes at four weeks relative to } \\
\text { baseline (mean } 20 \mathrm{SE} \pm 3 \vee 3 \pm 1, \mathrm{P}=0.01 \text { ) }\end{array}$ \\
\hline De Vries $(2007)^{94}$ & 24 & $\begin{array}{l}\text { TENS/spinal cord } \\
\text { stimulation }\end{array}$ & $\begin{array}{l}\text { Refractory angina, } \\
\text { NOCA }\end{array}$ & Not assessed & $\begin{array}{l}\text { Non-randomised } \\
\text { intervention }\end{array}$ & $\begin{array}{l}\text { TENS, followed by spinal cord stimulator, if TENS caused skin } \\
\text { irritation, improved SAQ angina frequency (mean } 42 \mathrm{SD} \pm 24 \text { to } \\
55 \pm 23 ; \mathrm{P}=0.005 \text { ) }\end{array}$ \\
\hline Luo $(2012)^{95}$ & 45 & EECP & Angina, NCA & Abnormal CFR & $\begin{array}{l}\text { Non-randomised } \\
\text { intervention }\end{array}$ & $\begin{array}{l}\text { EECP improved CCS angina class at eight weeks, relative to } \\
\text { baseline (mean } 2.96 \mathrm{SD} \pm 0.69 \text { to } 1.75 \pm 0.85, P<0.001 \text { ). }\end{array}$ \\
\hline
\end{tabular}

NOCA=non-obstructive coronary arteries: $\mathrm{NCA}=$ normal coronary arteries: $\mathrm{STE}=\mathrm{ST}$ segment elevation; $\mathrm{RCT}=$ randomised clinical trial; $\mathrm{CFR}=\mathrm{coronary}$ flow reserve; $\mathrm{CSFP}=\mathrm{Coronary}$ slow flow phenomenon; $\mathrm{SAQ}=$ Seattle Angina Questionnaire; WO=women only; $C C B=$ calcium channel blockers; $C C S=C a n a d i a n$ Cardiovascular Society; TENS=transcutaneous electrical nerve stimulation; $\mathrm{EECP}=$ enhanced external counterpulsation; $\mathrm{ECG}=$ electrocardiogram; $\mathrm{SD}=$ standard deviation; $\mathrm{SE}=$ standard error. 


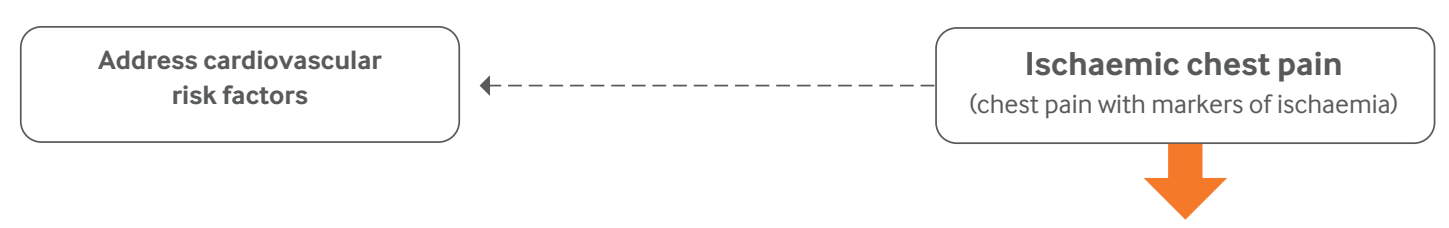

Obstructive coronary arteries (epicardial coronary lesions $\geq 50 \%$ )
Structural coronary angiography

(invasive or coronary CT angiography) Non-obstructive coronary arteries ( $<50 \%$ lesions)
Non-cardiac causes Non-ischaemic causes
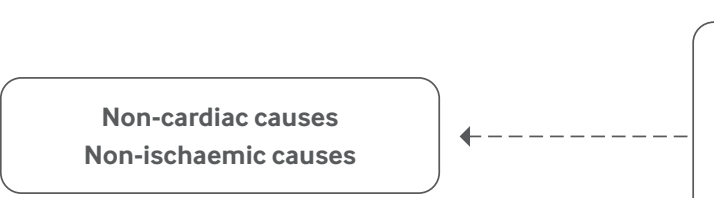

Functional coronary angiography

- Exclude obstructive coronary lesions - fractional flow reserve

- Coronary macrovascular dysfunction - acetylcholine provocative spasm test

- Coronary microvascular dysfunction - coronary flow reserve, index of microcirculatory resistance or hyperaemic microvascular resistance
Avoid precipitants

Cardioprotective agents

Anti-ischaemic agents and treatments

Monitor status

Refractory angina

- Other anti-ischaemic treatments

- Anti-nociceptive agents

\section{Coronary macrovascular dysfunction} (vasospastic angina)

- Smoking, sympathomimetics, $\beta$ blockers

- Calcium channel blockers

- Nitrates, calcium channel blockers, potassium channel activator, phosphodiesterase-III inhibitor, statin, Rho kinase inhibitor

- Exercise training or cardiac rehabilitation

- Health status (symptoms) or major adverse cardiovascular events

- Stellate ganglion block

- Analgesics
Coronary microvascular dysfunction (coronary microvascular disorders)

Stress?

- Unclear - statin? Angiotensin converting enzyme inhibitor?

- Calcium channel blockers, potassium channel activator, nitrates, statin, Rho kinase inhibitor, $\beta$ blockers, sinus node inhibitor, late sodium current blocker, fatty acid oxidation inhibitor, angiotensin converting enzyme inhibitor; consider mechanism, angina, and comorbidities

- Exercise training or cardiac rehabilitation

- Health status (symptoms) or major adverse cardiovascular events

- External enhanced counterpulsation, coronary sinus reducer

- Antidepressants, methylxanthines, analgesics, transcutaneous electrical nerve stimulator, spinal stimulators

Fig 5 | How to treat patients with INOCA-a personalised medicine approach. CT=computed tomography

in women. Patients with microvascular angina can experience rest angina, atypical symptoms, or episodes triggered by emotional stress. ${ }^{110}$ While the classic clinical presentations might be useful in delineating the pathophysiological syndromes, functional coronary angiography is a more objective modality.

\section{Avoid precipitating and aggravating factors Smoking}

Except for smoking, the conventional cardiovascular risk factors are less prevalent in patients with INOCA than in those with obstructive coronary artery disease. However, smoking is a key risk factor for vasospastic angina, ${ }^{109}$ where its cessation is strongly recommended.

\section{Sympathomimetic agents}

These agents include the recreational use of cocaine, ${ }^{111}$ methamphetamines (ice), ${ }^{112}$ and methylenedioxy-N-methamphetamine (ecstasy), ${ }^{113}$ all of which could precipitate vasospastic angina episodes. In addition, therapeutically used sympathomimetic agents (adrenaline, ${ }^{114}$ 
noradrenaline, and methylphenidate ${ }^{115}$ ) have been associated with precipitating episodes of coronary spasm.

\section{Beta blockers}

These agents have been reported to precipitate episodes of vasospastic angina by augmenting unopposed catecholamine alpha constrictor effects, and thus should be avoided in these patients. ${ }^{116}$ In contrast, beta blockers have been reported to improve angina in patients with coronary microvascular dysfunction $^{35}$; reflecting the importance in delineating the different pathophysiological mechanisms.

\section{Other drug treatments}

Ergot alkaloids ${ }^{117}$ and serotonin ${ }^{118}$ have been used as provocative stimuli in spasm testing. Hence ergot preparations used in the treatment of migraine ${ }^{119}$ or uterine bleeding ${ }^{120}$ and serotonin uptake inhibitors used in treating depression ${ }^{121}$ could precipitate coronary spasm. Some chemotherapy agents (5-flurouracil, ${ }^{122}$ capecitabine, ${ }^{123}$ sorafenib ${ }^{124}$ ) have also been described to precipitate episodes of vasospastic angina. General anaesthesia has been associated with inducing coronary spasm, ${ }^{125}$ although the multiple agents used make it difficult to discern which particular agents are involved. Accordingly, the above drug treatments should be avoided or used with caution in patients with vasospastic angina. Their impact on coronary microvascular disorders is less clear.

\section{Mental stress}

Pathophysiological studies have shown that mental stress could provoke epicardial coronary artery constriction, ${ }^{126}$ coronary microvascular dysfunction, ${ }^{127}$ myocardial ischaemia, ${ }^{128} 129$ and angina $^{127}$ in the absence of obstructive coronary artery disease. These findings might explain why many patients with INOCA report that mental stress precipitates or aggravates their symptoms. In addition, clinical studies have reported an association between INOCA and anxiety disorders, ${ }^{130-133}$ as well as depression. ${ }^{131} 132$ What is unknown is whether these psychiatric disorders predispose to INOCA or the suboptimal management of INOCA precipitates these disorders. Regardless, mental stress should be avoided or minimised in patients with INOCA.

\section{Cardioprotective treatments \\ Vasospastic angina}

Calcium channel blockers are considered a key treatment for vasospastic angina because they have been shown to be an independent determinant of infarct-free survival, ${ }^{37}$ and their cessation is associated with an increased risk of MACE. ${ }^{21}$ As for other anti-vasospastic agents (such as nitrates or nicorandil), evidence to support their routine use as cardioprotective agents is insufficient, in addition to concerns that chronic nitrate use might increase MACE owing to nitrate tolerance. ${ }^{134}$ Furthermore, implantable cardioverter defibrillators as a sudden death preventive measure might be warranted for patients with vasospastic angina who present with cardiac arrest or malignant arrhythmias. No randomised trials have examined the impact of implantable cardioverter defibrillators in this scenario, and small observational studies have yielded conflicting data. ${ }^{135-137}$

\section{Coronary microvascular disorders}

Advice for coronary microvascular disorders is more challenging because no clinical trial data exist to support the use of cardioprotective agents, although statins and angiotensin converting enzyme inhibitors are often recommended in clinical guidelines, ${ }^{108}$ and these treatments are the focus of a large prospective clinical trial that is in progress. ${ }^{138}$

\section{Anti-anginal treatments}

Vasospastic angina

In addition to cardioprotective benefits, calcium channel blockers are effective at reducing angina symptoms in these patients. ${ }^{21}$ Long acting nitrates have also been traditionally used in the anti-anginal management of vasospastic angina, especially given that the pain is responsive to nitrates ${ }^{21}$; however, as stated above, their benefits have recently been questioned. $^{134}$ Other novel agents with clinical randomised controlled trial data supporting their use as anti-anginal agents in vasospastic angina include nicorandil ${ }^{41}{ }^{42}$ and cilostazol $^{57}$ (table 3). In addition, aerobic exercise training has been shown to reduce angina episodes in patients with vasospastic angina..$^{55}$

\section{Coronary microvascular disorders}

The use of anti-anginal treatments in coronary microvascular disorders is more complex considering the multiple pathophysiological mechanisms, clinical subtypes, and heterogeneity in trial results. Nitrates, for example, could further impair coronary blood flow ${ }^{63}$ and aggravate symptoms in patients with these disorders. Consequently, unlike vasospastic angina, no uniform first line treatment exists. Table 4 summarises the anti-anginal clinical trial evidence base currently available. The choice of agent will need to be based on clinical assessment as to:

- The suspected underlying mechanismischaemia (due to impaired vasodilation, resting constriction, or hyper-reactivity) versus hyperalgesia nociception (attributable to a defective pathway at the level of the receptor, afferent nerves, spinal cord, or higher centres)

- The angina pattern-exertional angina (where heart rate reduction might be beneficial), rest angina (where coronary microvascular vasodilators might be more beneficial), or mixed pattern angina (that is, exertional and rest angina episodes)

- $\quad$ The concurrent disorders (eg, asthma, where $\beta$ blockers are contraindicated). 
In contrast to pharmacological anti-anginal treatments, exercise training, cardiac rehabilitation, and relaxation therapy have consistently shown benefit in coronary microvascular disorders (table 4). Such benefits include improved angina control ${ }^{85} 87$ and increased exercise capacity. ${ }^{88-90}$

\section{When to treat?}

With knowledge of the INOCA treatment targets, the heterogeneous patient population, and how to tailor treatments to individual patients (personalised medicine), it is important to consider when is best to initiate the various treatments (fig 1). This approach will also need to be personalised, depending on the patient's progress.

\section{Initial diagnosis}

The diagnosis of INOCA requires the documentation of non-obstructive coronary arteries; therefore, it is most frequently diagnosedimmediately after coronary angiography. In patients with vasospastic angina, calcium channel blockers should be immediately commenced, owing to their cardioprotective effects, although no definitive evidence exists for the benefits of cardioprotective agents in coronary microvascular disorders. Initiating anti-anginal treatment in patients with INOCA after diagnostic angiography will depend on the patients' symptomatic status, which will vary according to the treating clinician's threshold for performing angiography. For individuals with infrequent mild symptoms, the clinician and patient could decide to observe the patient's progress, using only short acting nitrates during acute episodes, before initiating regular maintenance anti-anginal treatments. However, for patients with more disabling pain, anti-anginal treatments should be promptly initiated, targeting the underlying pathophysiological mechanisms. As a guide to when to commence anti-anginal treatments, the angina frequency can provide a useful clinical surrogate.

As shown in figu 6, a linear relation exists between angina frequency and other markers of health status (physical limitation and quality of life), with a substantial step between monthly and weekly angina episodes. Hence a potential clinical threshold for considering when to initiate anti-anginal treatments is when angina episodes occur at least once a week. However, patients will differ in their perception of the angina symptoms, so treatment decisions should be personalised and considered via a shared decision making process.

\section{Clinical monitoring of health status}

Patients with vasospastic angina have long been recognised to have so-called hot phases, when the angina episodes are recalcitrant and difficult to manage, as well as so-called cold phases, when the disorder is quiescent. ${ }^{109}$ This fluctuating pattern is likely to represent periodic changes in the underlying pathophysiology and precipitants. Although not as clearly documented, this pattern might also occur in coronary microvascular disorders. Because the anti-anginal treatments are targeted towards INOCA symptoms, there could be quiescent periods when these treatments can be cautiously scaled back.

When following up patients, clinicians should be aware that they often overestimate the efficacy of their treatments. In chronic stable angina studies, clinicians frequently perceived the patient's angina as being optimally controlled, whereas the patient's perception was not as positive. ${ }^{34}$ This discordance in perception is attributable to clinicians underestimating the angina burden, because $42 \%$ of patients report more angina than noted by their clinicians. ${ }^{139}$ Variation in clinician skills for obtaining an angina history might account for these differences and could be minimised by a standardised angina collection tool to optimise clinical care. ${ }^{139}$

\section{Managing refractory angina}

Patients with INOCA who have refractory angina (that is, who are unresponsive to two or more antianginal treatments) are likely to need particularly challenging clinical management. Firstly, doctors should consider whether the patient has developed obstructive coronary artery disease, which opens up more therapeutic options (that is, revascularisation treatments). Secondly, functional coronary angiography should be considered if not already performed, because the confirmation of vasospastic angina would guide clinicians to the more aggressive use of specific anti-anginal treatments. For example, case reports of high dose calcium channel blockers in patients with vasospastic angina have reported considerable benefits. ${ }^{140} 141$ Thirdly, patients who have a suboptimal response to conventional pharmacological anti-ischaemic agents warrant consideration for anti-nociceptive and non-pharmacological treatments (tables 2 and 3). Initiating these treatments will be based on the patient's health status and should be undertaken in a shared decision making model of care, especially considering the limited evidence base

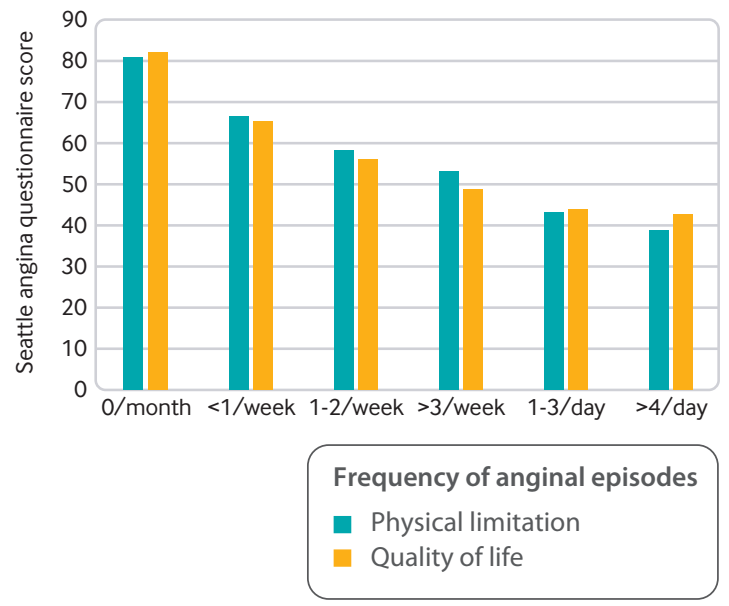

Fig 6 | Associations between angina frequency, physical limitations, and quality of life. Data taken from Beltrame et $\mathrm{al}^{34}$ 
available. Moreover, a care plan is often useful in patients who frequently present to hospital with uncontrolled angina to facilitate prompt initiation of appropriate treatment and avoid misplaced management approaches by hospital staff unfamiliar with the patient. In addition, involvement of a pain management unit will be beneficial for patients with chronic symptoms. These multidisciplinary teams have extensive experience in managing refractory pain syndromes and have access to valuable resources such as specialised clinical psychologists or psychiatrists, as well as multiple anti-nociceptive treatments (table 2).

\section{Where to next-emerging treatments}

Although calcium channel blockers have been well established as the first line agent in vasospastic angina, the optimal first-line treatment for coronary microvascular disorders remains elusive. Contemporary clinical trial approaches have used pharmacological treatments and novel treatment strategies.

These emerging treatments will provide a broader array of tools in managing angina secondary to epicardial coronary spasm or coronary microvascular dysfunction. Although study results of these important innovations are yet to come, the progress of many studies has been delayed by the covid-19 pandemic.

\section{Intense medical treatment}

Intense medical treatment (that is, high intensity statins plus angiotensin converting enzyme inhibitors or angiotensin receptor blockers) could reduce MACE in patients with INOCA. The WARRIOR trial is a multicentre study with a PROBE design (prospective, randomised, open-label with blindedendpoint analysis) comparing intensive medical treatment with usual care in patients with INOCA, evaluating MACE (death, non-fatal myocardial infarction or stroke, or hospital admission for angina or heart failure) at an average follow-up period of three years. ${ }^{138}$ Study completion is anticipated within the next few years when it will provide important insights into the potential benefits of conventional cardioprotective treatments in INOCA.

\section{Ticagrelor}

The TIC (ticagrelor in coronary microvascular dysfunction) trial (Australian and New Zealand Clinical Trials ACTRN12616000388415) evaluates the potential anti-anginal benefits of this anti-platelet agent in the coronary slow flow phenomenon. Ticagrelor inhibits erythrocyte adenosine uptake, thereby increasing plasma adenosine levels, which inhibits purinergic $2\left(\mathrm{P}_{2}\right)$ platelet receptors to exert an anti-platelet effect. The raised endogenous adenosine levels also stimulate the purinergic $1\left(\mathrm{P}_{1}\right)$ receptors, which includes the $A_{2 A}$ receptor, resulting in coronary microvascular vasodilation. The TIC trial uses a randomised, double blind, placebo controlled crossover design to examine the anti-anginal effects of ticagrelor (90 mg twice daily) in patients with symptoms with the coronary slow flow phenomenon. The study findings should be available in two years and potentially provide another anti-anginal strategy for coronary microvascular disorders.

\section{Endothelin receptor blockers}

Endothelin receptor blockers could be of benefit in coronary microvascular disorders because endothelin is a potent coronary microvascular constrictor. Zibotentan is a specific endothelin A receptor antagonist and is being examined in two contemporary randomised clinical trials. The first trial (ACTRN12618000021279) uses the same design as the TIC trial (see above) to evaluate the anti-anginal effects of zibotentan (10 mg daily) in patients with symptoms with the coronary slow flow phenomenon. The second trial (the PRIZE (precision medicine with zibotentan in microvascular angina) trial; ClinicalTrials.gov NCT04097314) uses a randomised, double blind, placebo controlled crossover design to examine the impact of zibotentan (10 mg daily) on treadmill exercise time in patients with microvascular angina. Results from both trials should be available within two years.

\section{Rhodiola rosea}

Rhodiola rosea is a traditional herbal medicine that has multiple pharmacological properties attributed to its major active ingredient, salidroside. These properties include anti-oxidant and antiinflammatory effects, but of particular relevance to patients with INOCA is its ability to block calcium channels in vascular smooth muscle cells. ${ }^{142}$ Accordingly, a randomised, double blind, placebo controlled trial is in progress to assess the impact of Rhodiola rosea capsules on coronary flow reserve and symptoms, in patients with INOCA symptoms with an impaired coronary flow reserve (NCT04218916). The efficacy of this herbal medicine should be evident in the next two years.

\section{Autologous CD34 stem cells}

Autologous CD34 stem cells administration has been shown to improve treadmill exercise time and angina frequency in randomised, double blind, placebo controlled trials among patients with obstructive coronary artery disease. ${ }^{143}$ This benefit is attributable to the stem cells differentiating into endothelial cells and potentially releasing angiogenic cytokines that aid microvascular recovery. Moreover, a preliminary feasibility study in patients with INOCA suggests that they might also benefit from this treatment. ${ }^{144}$ Based on these findings, the FREEDOM study has been initiated (NCT04614467); a randomised double blind, placebo controlled study evaluating the effect of one intracoronary administration of autologous CD34 stem cells on coronary flow reserve and angina frequency. The results of this novel strategy should be available in the next two years and will affect future therapeutic approaches in patients with INOCA. 


\section{Coronary sinus reducer}

The coronary sinus reducer (Reducer, Neovasc) is balloon expandable, stainless steel, hourglass shaped endoluminal device, which is percutaneously implanted into the coronary sinus to increase coronary venous pressure thereby reducing coronary microvascular resistance. A previous clinical trial in patients with refractory angina and obstructive coronary artery disease has confirmed its benefit in alleviating refractory angina symptoms, ${ }^{145}$ as has a recent preliminary study in patients with INOCA. ${ }^{146}$ Within two to three years, the open label COSIMA trial (NCT04606459) will be completed, which compares the impact of device implantation and medical treatment on angina status in patients with INOCA. If effective, this single procedure, percutaneous intervention could provide another tool for the management of angina in patients with INOCA.

\section{Guidelines}

No professional society guidelines exclusively focus on INOCA, but the European Association of Percutaneous Cardiovascular Interventions, in collaboration with European Society of Cardiology (ESC) and COVADIS, have recently published a contemporary consensus document on INOCA. ${ }^{147}$ This consensus document complements the present review by elaborating on diagnostic approaches in INOCA.

The 2019 ESC guidelines on the diagnosis and management of chronic coronary syndromes ${ }^{17}$ have a section regarding INOCA. Specific guideline recommendations have been published in relation to the investigation of vasospastic angina and microvascular angina. For patients with suspected vasospastic angina, class I recommendations include an ECG during anginal episodes and coronary artery imaging (invasive angiography or coronary CT angiography) to determine the extent of underlying atherosclerotic disease. Class IIa recommendations for these patients include ambulatory ST-segment monitoring and intracoronary provocative spasm testing. ${ }^{17}$ For patients with suspected microvascular angina, a class IIa recommendation includes guidewire based, invasive coronary flow reserve or microvascular resistance measures in patients with non-obstructive coronary arteries. Class IIb recommendations include intracoronary acetylcholine assessment for microvascular spasm or non-invasive coronary flow reserve assessment via echocardiographic Doppler, cardiac magnetic resonance imaging, or positron emission tomography. ${ }^{17}$

These guidelines and consensus statements reflect the increasing clinical interest in INOCA and the endorsement by professional societies of the importance of diagnosing these disorders.

\section{Conclusion}

This comprehensive review has discussed the key interrogative questions relating to patient management of INOCA (fig 1). Accordingly, along with other recent authoritative papers, ${ }^{1} 108147148$ it provides the current evidence base to facilitate the diagnosis and management of these often neglected disorders. The future challenge is to implement what we already know concerning the diagnosis of INOCA and to expand the effectiveness of available treatments by personalising their use to the individual patient endotype.

The methods required to diagnose INOCA are well established and strongly advocated for in recent consensus statements ${ }^{1}{ }^{147} 148$ and guidelines. ${ }^{108}$ However, even in specialised centres proficient with functional coronary angiography techniques, few patients $(<10 \%)$ with chest pain and non-obstructive coronary arteries undergo routine evaluation for a functional coronary cause of their symptoms. Therefore, change management principles are required to implement the more appropriate use of functional coronary angiography, considering that more than half of elective angiograms do not have obstructive coronary artery disease to account for their presenting symptoms. Achieving this practice change will require increasing awareness of these disorders by the continued advocacy of professional cardiology societies and dedicated medical societies (eg, COVADIS, https://covadis. online/), as well as consumer involvement via patient support groups (eg, INOCA International, https: / / www.inocainternational.com/; International Heart Spasm Alliance, https://www. internationalheartspasmsalliance.org/).

Implementation of routine functional coronary angiography in patients with INOCA will also encourage personalised treatment. INOCA is a heterogeneous disorder and the importance of delineating vasospastic angina and coronary microvascular disorders has been described. However, coronary microvascular disorders have multiple mechanisms, including different coronary pathophysiology producing myocardial ischaemia, as well as disturbances in nociception. With well characterised cohorts of patients with INOCA (that is, clinical and pathophysiological characteristics), the individual treatments that patients are more

\section{PATIENT INVOLVEMENT}

The manuscript was provided to two female patients with coronary vasomotor disorders who founded the International Heart Spasm Alliance. The patients expressed the need for earlier diagnosis and more recognition in the medical community and advocated for a holistic approach to assessment and management. Based on their reviews, the manuscript was modified to emphasise challenges and opportunities in current practice, such as the lack of clinical trial data for cardioprotective agents, minimising the impact on mental wellbeing, the usefulness of care plans for recurrent hospital admissions, and the role of multidisciplinary approaches including exercise training and chronic pain management. 


\section{Questions for future research}

- Do cardioprotective treatments such as aspirin and statins reduce major adverse cardiovascular events in patients with ischaemia with non-obstructive coronary arteries (INOCA)?

- How can a standardised angina collection tool be implemented as a standard of care practice?

-What are the clinical outcomes of different INOCA subtypes?Contributors: DJ, RT, CZ, and JB conducted a review of the literature and prepared the manuscript draft. All authors were substantially involved in the conception, drafting, and editing of the manuscript. All authors have given final approval of the manuscript and are accountable for all portions of the manuscript. JB is the guarantor.

likely to respond to can be identified, which would be preferable to the trial-and-error approach currently used by clinicians. It would also provide a productive environment for the assessment of new INOCA treatments to improve the outcomes of these disorders.

Competing interests: We have read and understood the BMJ policy on declaration of interests and declare the following interests: none.

Provenance and peer review: Commissioned; externally peer reviewed

1 Bairey Merz CN, Pepine CJ, Walsh MN, Fleg JL. Ischemia and No Obstructive Coronary Artery Disease (INOCA): Developing evidencebased therapies and research agenda for the next decade. Circulation 2017;135:1075-92. doi:10.1161/CIRCULATIONAHA.116.024534

2 Patel MR, Peterson ED, Dai D, et al. Low diagnostic yield of elective coronary angiography. N Engl J Med 2010;362:886-95. doi:10.1056/NEJMoa0907272

3 Radico F, Zimarino M, Fulgenzi F, et al. Determinants of long-term clinical outcomes in patients with angina but without obstructive coronary artery disease: a systematic review and meta-analysis. Eur Heart / 2018;39:2135-46. doi:10.1093/eurheartj/ehy185

4 Jespersen L, Abildstrom SZ, Hvelplund A, et al. Burden of hospital admission and repeat angiography in angina pectoris patients with and without coronary artery disease: a registry-based cohort study. PLoS One 2014;9:e93170. doi:10.1371/journal.pone.0093170

5 Shaw LJ, Merz CN, Pepine Cl, et al, Women's Ischemia Syndrome Evaluation (WISE) Investigators. The economic burden of angina in women with suspected ischemic heart disease: results from the National Institutes of Health--National Heart, Lung, and Blood Institute--sponsored Women's Ischemia Syndrome Evaluation. Circulation 2006;114:894-904. doi:10.1161/ CIRCULATIONAHA.105.609990

6 Takagi Y, Takahashi J, Yasuda S, et al, Japanese Coronary Spasm Association. Prognostic stratification of patients with vasospastic angina: a comprehensive clinical risk score developed by the Japanese Coronary Spasm Association. J Am Coll Cardiol 2013;62:1144-53. doi:10.1016/j.jacc.2013.07.018

7 Sato K, Takahashi J, Odaka Y, et al, Japanese Coronary Spasm Association. Clinical characteristics and long-term prognosis of contemporary patients with vasospastic angina: Ethnic differences detected in an international comparative study. Int J Cardiol 2019:291:13-8. doi:10.1016/j.ijcard.2019.02.038

8 Shimokawa H, Suda A, Takahashi J, et al. Clinical characteristics and prognosis of patients with microvascular angina: an international and prospective cohort study by the Coronary Vasomotor Disorders International Study (COVADIS) Group. Eur Heart J 2021; ehab282. doi:10.1093/eurheartj/ehab282

9 Rumsfeld JS. Health status and clinical practice: when will they meet? Circulation 2002;106:5-7. doi:10.1161/01. CIR.0000020805.31531.48

10 Spertus JA. Evolving applications for patient-centered health status measures. Circulation 2008;118:2103-10. doi:10.1161/ CIRCULATIONAHA.107.747568

11 Di Fiore DP, Beltrame JF. Chest pain in patients with "normal angiography': could it be cardiac? Int J Evid Based Healthc 2013:11:56-68. doi:10.1111/1744-1609.12002

12 Tavella R, Cutri N, Tucker G, Adams R, Spertus J, Beltrame JF. Natural history of patients with insignificant coronary artery disease. Eur
HeartJ Qual Care Clin Outcomes 2016;2:117-24. doi:10.1093/ ehjicco/qcv034

13 Ford TJ, Stanley B, Good R, et al. Stratified Medical Therapy Using Invasive Coronary Function Testing in Angina: The CorMicA Trial. J Am Coll Cardiol 2018;72(23 Pt A):2841-55. doi:10.1016/j. jacc.2018.09.006

14 Ford TJ, Stanley B, Sidik N, et al. 1-Year Outcomes of Angina Management Guided by Invasive Coronary Function Testing (CorMicA). JACC Cardiovasc Interv 2020;13:33-45. doi:10.1016/j. jcin.2019.11.001

15 Lee BK, Lim HS, Fearon WF, et al. Invasive evaluation of patients with angina in the absence of obstructive coronary artery disease. Circulation 2015;131:1054-60. doi:10.1161/ CIRCULATIONAHA.114.012636

16 Sheikh AR, Zeitz C, Beltrame JF. Letter by Sheikh et al regarding article, "Invasive evaluation of patients with angina in the absence of pbstructive coronary artery disease". Circulation 2015;132:e242. doi:10.1161/CIRCULATIONAHA.115.017254

17 Knuuti J, Wiins W, Saraste A et al, ESC Scientific Document Group. 2019 ESC Guidelines for the diagnosis and management of chronic coronary syndromes. Eur Heart J 2020;41:407-77. doi:10.1093/ eurhearti/ehz425

18 Heberden W. Some account of a disorder of the breast. Medical Transactions. 1772;2:59-67.

19 Prinzmetal M, Kennamer R, Merliss R, Wada T, Bor N. Angina pectoris. I. A variant form of angina pectoris; preliminary report. Am J Med 1959;27:375-88. doi:10.1016/0002-9343(59)90003-8

20 Beltrame JF, Crea F, Kaski JC, et al, Coronary Vasomotion Disorders International Study Group (COVADIS). International standardization of diagnostic criteria for vasospastic angina. Eur Heart J 2017;38:2565-8

21 Beltrame JF, Crea F, Kaski JC, et al, Coronary Vasomotion Disorders International Study Group (COVADIS). The Who, What, Why, When, How and Where of Vasospastic Angina. Circ J 2016;80:289-98. doi:10.1253/circj.CJ-15-1202

22 Shimokawa H. 2014 Williams Harvey Lecture: importance of coronary vasomotion abnormalities-from bench to bedside. Eur Heart) 2014;35:3180-93. doi:10.1093/eurheartj/ehu427

23 Ong P, Aziz A, Hansen HS, Prescott E, Athanasiadis A, Sechtem $U$. Structural and functional coronary artery abnormalities in patients with vasospastic angina pectoris. Circ J 2015;79:1431-8. doi:10.1253/circj.CJ-15-0520

24 Beltrame JF, Crea F, Camici P. Advances in coronary microvascular dysfunction. Heart Lung Circ 2009;18:19-27. doi:10.1016/j. hlc.2008.11.002

25 Mohri M, Koyanagi M, Egashira K, et al. Angina pectoris caused by coronary microvascular spasm. Lancet 1998;351:1165-9. doi:10.1016/S0140-6736(97)07329-7

26 Opherk D, Zebe H, Weihe E, et al. Reduced coronary dilatory capacity and ultrastructural changes of the myocardium in patients with angina pectoris but normal coronary arteriograms. Circulation 1981;63:817-25. doi:10.1161/01.CIR.63.4.817

27 Beltrame JF, Crea F, Kaski JC, et al, Coronary Vasomotion Disorders International Study Group (COVADIS). International standardization of diagnostic criteria for vasospastic angina. Eur Heart/ 2017;38:2565-8.

$28 \mathrm{Kemp} \mathrm{HGJr}$. Left ventricular function in patients with the anginal syndrome and normal coronary arteriograms. Am J Cardiol 1973;32:375-6. doi:10.1016/S0002-9149(73)80150-X

29 Cannon RO3rd, Epstein SE. "Microvascular angina" as a cause of chest pain with angiographically normal coronary arteries. Am J Cardiol 1988;61:1338-43. doi:10.1016/0002-9149(88)91180-0

30 Ong P, Camici PG, Beltrame JF, et al, Coronary Vasomotion Disorders International Study Group (COVADIS). International standardization of diagnostic criteria for microvascular angina. Int J Cardiol 2018;250:16-20. doi:10.1016/j.ijcard.2017.08.068

31 Beltrame JF. Defining the coronary slow flow phenomenon. Circ I 2012;76:818-20. doi:10.1253/circj.CJ-12-0205

32 Beltrame JF, Limaye SB, Horowitz JD. The coronary slow flow phenomenon--a new coronary microvascular disorder. Cardiology 2002;97:197-202. doi:10.1159/000063121

33 Ong P, Athanasiadis A, Borgulya G, Mahrholdt H, Kaski JC, Sechtem U. High prevalence of a pathological response to acetylcholine testing in patients with stable angina pectoris and unobstructed coronary arteries. The ACOVA Study (Abnormal COronary VAsomotion in patients with stable angina and unobstructed coronary arteries). J Am Coll Cardiol 2012;59:655-62. doi:10.1016/j.jacc.2011.11.015

34 Beltrame JF, Weekes AJ, Morgan C, Tavella R, Spertus JA. The prevalence of weekly angina among patients with chronic stable angina in primary care practices: The Coronary Artery Disease in General Practice (CADENCE) Study. Arch Intern Med 2009;169:1491 9. doi:10.1001/archinternmed.2009.295

35 Lanza GA, Colonna G, Pasceri V, Maseri A. Atenolol versus amlodipine versus isosorbide-5-mononitrate on anginal symptoms in syndrome X. Am J Cardiol 1999;84:854-6, A8. doi:10.1016/S00029149(99)00450-6 
36 Lanza GA, Manzoli A, Bia E, Crea F, Maseri A. Acute effects of nitrates on exercise testing in patients with syndrome $X$. Clinical and pathophysiological implications. Circulation 1994;90:2695-700. doi:10.1161/01.CIR.90.6.2695

37 Yasue H, Takizawa A, Nagao M, et al. Long-term prognosis for patients with variant angina and influential factors. Circulation 1988;78:1-9. doi:10.1161/01.CIR.78.1.1

38 Mehta PK, Goykhman P, Thomson LE, et al. Ranolazine improves angina in women with evidence of myocardial ischemia but no obstructive coronary artery disease. JACC Cardiovasc Imaging 2011;4:514-22. doi:10.1016/j.jcmg.2011.03.007

39 Villano A, Di Franco A, Nerla R, et al. Effects of ivabradine and ranolazine in patients with microvascular angina pectoris. Am J Cardiol 2013;112:8-13. doi:10.1016/j.amjcard.2013.02.045

40 Chen JW, Lee WL, Hsu NW, et al. Effects of short-term treatment of nicorandil on exercise-induced myocardial ischemia and abnormal cardiac autonomic activity in microvascular angina. Am J Cardiol 1997;80:32-8. doi:10.1016/S0002-9149(97)00279-8

41 Kishida H, Murao S. Effect of a new coronary vasodilator, nicorandil, on variant angina pectoris. Clin Pharmacol Ther 1987;42:166-74. doi:10.1038/clpt.1987.127

42 Lablanche JM, Bauters C, Leroy F, Bertrand ME. Prevention of coronary spasm by nicorandil: comparison with nifedipine. J Cardiovasc Pharmacol 1992;20(Suppl 3):S82-5. doi:10.1097/00005344199206203-00014

43 Huckell VF, McLaughlin PR, Morch JE, Wigle ED, Adelman AG. Prinzmetal's angina with documented coronary artery spasm. Treatment and follow-up. Br Heart J 1981;45:649-55. doi:10.1136/ hrt.45.6.649

44 Nalbantgil S, Altintiğ A, Yilmaz H, Nalbantgil I, Önder R. The effect of trimetazidine in the treatment of microvascular angina. Int J Angiol 1999:8:40-3. doi:10.1007/BF01616842

45 Heupler FAJr, Proudfit WL. Nifedipine therapy for refractory coronary arterial spasm. Am J Cardiol 1979;44:798-803. doi:10.1016/0002 9149(79)90200-5

46 Aschermann M, Bultas J, Karetová D, Kölbel F, Kozáková M Simper D. Randomized double-blind comparison of isosorbide dinitrate and nifedipine in variant angina pectoris. Am J Cardiol 1990;65:46J-9J. doi:10.1016/0002-9149(90)91312-

47 Chahine RA, Feldman RL, Giles TD, et al. Randomized placebocontrolled trial of amlodipine in vasospastic angina. Amlodipine Study 160 Group. I Am Coll Cardiol 1993;21:1365-70. doi:10.1016/0735-1097(93)90310-W

48 Higuma T, Oikawa K, Kato T, et al. Comparison of the effects of long-acting nifedipine CR and diltiazem $\mathrm{R}$ in patients with vasospastic angina: Aomori coronary spastic angina study. J Cardiol 2010;56:354-60. doi:10.1016/j.jjcc.2010.07.010

49 Oikawa Y, Matsuno S, Yajima J, et al. Effects of treatment with once-daily nifedipine CR and twice-daily benidipine on prevention of symptomatic attacks in patients with coronary spastic angina pectoris-Adalat Trial vs Coniel in Tokyo against Coronary Spastic Angina (ATTACK CSA). J Cardiol 2010;55:238-47. doi:10.1016/j.jjcc.2009.11.005

50 Kook H, Hong SJ, Yang KS, Lee S, Kim JS, Park CG. Comparison of nebivolol versus diltiazem in improving coronary artery spasm and quality of life in patients with hypertension and vasospastic angina: A prospective, randomized, double-blind pilot study. PLoS One 2020;15:e0239039. doi:10.1371/journal.pone.0239039

51 Shimizu H, Lee JD, Ogawa KB, et al. Efficacy of denopamine, a beta 1 adrenoceptor agonist, in preventing coronary artery spasm. Jpn Circ 1993:57:175-82. doi:10.1253/jcj.57.175

52 Yasue H, Mizuno Y, Harada E, et al, SCAST (Statin and Coronary Artery Spasm Trial) Investigators. Effects of a 3-hydroxy-3-methylglutaryl coenzyme A reductase inhibitor, fluvastatin, on coronary spasm after withdrawal of calcium-channel blockers. J Am Coll Cardiol 2008;51:1742-8. doi:10.1016/j.jacc.2007.12.049

53 Kim SR, Choi KH, Song YB, et al. Effect of sarpogrelate and highdose statin on the reduction of coronary spasm in vasospastic angina: A two by two factorial, pilot randomized study. Clin Cardiol 2019;42:899-907. doi:10.1002/clc.23239

54 Kawano H, Motoyama T, Hirai N, Kugiyama K, Ogawa H, Yasue H. Estradiol supplementation suppresses hyperventilation-induced attacks in postmenopausal women with variant angina. J Am Coll Cardiol 2001;37:735-40. doi:10.1016/S0735-1097(00)01187-6

55 Morikawa Y, Mizuno Y, Harada E, et al. Aerobic interval exercise training in the afternoon reduces attacks of coronary spastic angina in conjunction with improvement in endothelial function, oxidative stress, and inflammation. Coron Artery Dis 2013;24:177-82. doi:10.1097/MCA.0b013e32835cbef5

56 Morita S, Mizuno Y, Harada E, et al. Pioglitazone, a peroxisome proliferator-activated receptor $\gamma$ activator, suppresses coronary spasm. Coron Artery Dis 2014;25:671-7. doi:10.1097/ MCA.0000000000000144

57 Shin ES, Lee JH, Yoo SY, et al. A randomised, multicentre, double blind, placebo controlled trial to evaluate the efficacy and safety of cilostazol in patients with vasospastic angina. Heart 2014:100:1531-6. doi:10.1136/heartinl-2014-305986

58 Cannon RO3rd, Watson RM, Rosing DR, Epstein SE. Efficacy of calcium channel blocker therapy for angina pectoris resulting from small-vessel coronary artery disease and abnormal vasodilator reserve. Am J Cardiol 1985;56:242-6. doi:10.1016/0002 9149(85)90842-2

59 Cannon RO3rd, Brush JEJr, Schenke WH, Tracy CM, Epstein SE. Beneficial and detrimental effects of lidoflazine in microvascular angina. Am J Cardiol 1990;66:37-41. doi:10.1016/00029149(90)90732-G

60 Beltrame JF, Turner SP, Leslie SL, Solomon P, Freedman SB, Horowitz JD. The angiographic and clinical benefits of mibefradil in the coronary slow flow phenomenon. J Am Coll Cardiol 2004;44:57-62. doi:10.1016/j.jacc.2004.03.055

61 Zhang X, Li Q, Zhao J, et al. Effects of combination of statin and calcium channel blocker in patients with cardiac syndrome X. Coron Artery Dis 2014;25:40-4. doi:10.1097/MCA.0000000000000054

62 Sen N, Tavil Y, Erdamar H, et al. Nebivolol therapy improves endothelial function and increases exercise tolerance in patients with cardiac syndrome X. Anadolu Kardiyol Derg 2009;9:371-9.

63 Bugiardini R, Borghi A, Pozzati A, Ottani F, Morgagni GL, Puddu $P$. The paradox of nitrates in patients with angina pectoris and angiographically normal coronary arteries. Am J Cardiol 1993;72:343-7. doi:10.1016/0002-9149(93)90683-4

64 Russo G, Di Franco A, Lamendola P, et al. Lack of effect of nitrates on exercise stress test results in patients with microvascular angina. Cardiovasc Drugs Ther 2013;27:229-34. doi:10.1007/s10557013-6439-z

65 Yamabe H, Namura H, Yano T, et al. Effect of nicorandil on abnormal coronary flow reserve assessed by exercise 201TI scintigraphy in patients with angina pectoris and nearly normal coronary arteriograms. Cardiovasc Drugs Ther 1995:9:755-61. doi:10.1007/ BF00879868

66 Kayikcioglu M, Payzin S, Yavuzgil O, Kultursay H, Can LH, Soydan I. Benefits of statin treatment in cardiac syndrome-X1. Eur Heart I 2003;24:1999-2005. doi:10.1016/S0195-668X(03)00478-0

67 Fábián E, Varga A, Picano E, Vajo Z, Rónaszéki A, Csanády M. Effect of simvastatin on endothelial function in cardiac syndrome X patients. Am / Cardiol 2004;94:652-5. doi:10.1016/j.amjcard.2004.05.035

68 Merz CN, Olson MB, McClure C, et al. A randomized controlled trial of low-dose hormone therapy on myocardial ischemia in postmenopausal women with no obstructive coronary artery disease: results from the National Institutes of Health/National Heart, Lung, and Blood Institute-sponsored Women's Ischemia Syndrome Evaluation (WISE). Am Heart J 2010;159:987. doi:10.1016/j. ahj.2010.03.024

69 Kaski JC, Rosano G, Gavrielides S, Chen L. Effects of angiotensinconverting enzyme inhibition on exercise-induced angina and ST segment depression in patients with microvascular angina. J Am Coll Cardiol 1994;23:652-7. doi:10.1016/0735-1097(94)90750-1

70 Motz W, Strauer BE. Improvement of coronary flow reserve after long-term therapy with enalapril. Hypertension 1996;27:1031-8. doi:10.1161/01.HYP.27.5.1031

71 Chen JW, Hsu NW, Wu TC, Lin SJ, Chang MS. Long-term angiotensin converting enzyme inhibition reduces plasma asymmetric dimethylarginine and improves endothelial nitric oxide bioavailability and coronary microvascular function in patients with syndrome X. Am I Cardiol 2002;90:974-82. doi:10.1016/S0002-9149(02)02664-4

72 Pauly DF, Johnson BD, Anderson RD, et al. In women with symptoms of cardiac ischemia, nonobstructive coronary arteries, and microvascular dysfunction, angiotensin-converting enzyme inhibition is associated with improved microvascular function: A doubleblind randomized study from the National Heart, Lung and Blood Institute Women's Ischemia Syndrome Evaluation (WISE). Am Heart 2011;162:678-84. doi:10.1016/j.ahj.2011.07.011

73 Bavry AA, Handberg EM, Huo T, et al. Aldosterone inhibition and coronary endothelial function in women without obstructive coronary artery disease: an ancillary study of the national heart, lung, and blood institute-sponsored women's ischemia syndrome evaluation. Am Heart J 2014;167:826-32. doi:10.1016/j.ahj.2014.01.017

74 Michelsen MM, Rask AB, Suhrs E, Raft KF, Høst N, Prescott E. Effect of ACE-inhibition on coronary microvascular function and symptoms in normotensive women with microvascular angina: A randomized placebo-controlled trial. PLoS One 2018;13:e0196962. doi:10.1371/journal.pone.0196962

75 Lerman A, Burnett JCJr, Higano ST, McKinley LJ, Holmes DRJr. Longterm L-arginine supplementation improves small-vessel coronary endothelial function in humans. Circulation 1998;97:2123-8. doi:10.1161/01.CIR 97.21.2123

76 Denardo SJ, Wen X, Handberg EM, et al. Effect of phosphodiesterase type 5 inhibition on microvascular coronary dysfunction in women: a Women's Ischemia Syndrome Evaluation (WISE) ancillary study. Clin Cardiol 2011;34:483-7. doi:10.1002/clc.20935 
77 Cannon RO3rd, Quyyumi AA, Mincemoyer R, et al. Imipramine in patients with chest pain despite normal coronary angiograms. N Engl J Med 1994:330:1411-7. doi:10.1056/NEIM199405193302003

78 Bøtker HE, Sonne HS, Schmitz O, Nielsen TT. Effects of doxazosin on exercise-induced angina pectoris, ST-segment depression, and insulin sensitivity in patients with syndrome X. Am / Cardiol 1998;82:1352-6. doi:10.1016/S0002-9149(98)00640-7

79 Rosen SD, Lorenzoni R, Kaski JC, Foale RA, Camici PG. Effect of alpha1-adrenoceptor blockade on coronary vasodilator reserve in cardiac syndrome X. J Cardiovasc Pharmacol 1999;34:554-60. doi:10.1097/00005344-199910000-00012

80 Topal E, Ozdemir R, Barutcu I, et al. The effects of trimetazidine on heart rate variability in patients with slow coronary artery flow. J Electrocardiol 2006;39:211-8. doi:10.1016/j. jelectrocard.2005.08.011

81 Bairey Merz CN, Handberg EM, Shufelt CL, et al. A randomized, placebo-controlled trial of late Na current inhibition (ranolazine) in coronary microvascular dysfunction (CMD): impact on angina and myocardial perfusion reserve. Eur Heart / 2016;37:1504-13. doi:10.1093/eurheartj/ehv647

82 Ahmed B, Mondragon J, Sheldon M, Clegg S. Impact of ranolazine on coronary microvascular dysfunction (MICRO) study. Cardiovasc Revasc Med 2017;18:431-5. doi:10.1016/i.carrev.2017.04.012

83 Rambarat CA, Elgendy IY, Handberg EM, et al. Late sodium channe blockade improves angina and myocardial perfusion in patients with severe coronary microvascular dysfunction: Women's Ischemia Syndrome Evaluation-Coronary Vascular Dysfunction ancillary study. Int J Cardiol 2019;276:8-13. doi:10.1016/j.ijcard.2018.09.081

84 Lim TK, Noman A, Choy AMJ, Khan F, Struthers AD, Lang CC. The APEX trial: Effects of allopurinol on exercise capacity, coronary and peripheral endothelial function, and natriuretic peptides in patients with cardiac syndrome X. Cardiovasc Ther 2018;36. doi:10.1111/1755-5922.12311

85 Eriksson BE, Tyni-Lennè R, Svedenhag J, et al. Physical training in Syndrome X: physical training counteracts deconditioning and pain in Syndrome X. J Am Coll Cardiol 2000;36:1619-25. doi:10.1016/ S0735-1097(00)00931-1

86 Tyni-Lenne R, Stryjan S, Eriksson B, Berglund M, Sylven C. Beneficial therapeutic effects of physical training and relaxation therapy in women with coronary syndrome X. Physiother Res Int 2002;7:35-43. doi:10.1002/pri.239

87 Asbury EA, Slattery C, Grant A, Evans L, Barbir M, Collins P. Cardiac rehabilitation for the treatment of women with chest pain and normal coronary arteries. Menopause 2008;15:454-60. doi:10.1097/ gme.0b013e31815982eb

88 Feizi A, Ghaderi C, Dehghani MR, Khalkhali HR, Sheikhi S. Effect of phase III cardiac rehabilitation and relaxation on the quality of life in patients with cardiac syndrome X. Iran J Nurs Midwifery Res 2012;17:547-52.

89 de Carvalho EE, Santi GL, Crescêncio JC, et al. Pilot study testing the effect of physical training over the myocardial perfusion and quality of life in patients with primary microvascular angina. J Nucl Cardiol 2015:22:130-7. doi:10.1007/s12350-014-9949-6

90 Szot W, Zając J, Kostkiewicz M, Owoc J, Bojar I. Cardiac rehabilitation: a good measure to improve quality of life in peri- and postmenopausal women with microvascular angina. Ann Agric Environ Med 2015;22:390-5. doi:10.5604/12321966.1152100

91 Bove KB, Nilsson M, Pedersen LR, et al. Comprehensive treatment of microvascular angina in overweight women - a randomized controlled pilot trial. PLoS One 2020;15:e0240722. doi:10.1371/ journal.pone.0240722

92 Eliasson T, Albertsson P, Hårdhammar P, Emanuelsson H, Augustinsson LE, Mannheimer C. Spinal cord stimulation in angina pectoris with normal coronary arteriograms. Coron Artery Dis 1993;4:819-27. doi:10.1097/00019501-199309000-00009

93 Jessurun GA, Hautvast RW, Tio RA, Delongste MJ. Electrical neuromodulation improves myocardial perfusion and ameliorates refractory angina pectoris in patients with syndrome $X$ : fad or future? Eur J Pain 2003;7:507-12. doi:10.1016/S1090-3801(03)00022-3

94 de Vries J, Dejongste MJ, Durenkamp A, Zijlstra F, Staal MJ. The sustained benefits of long-term neurostimulation in patients with refractory chest pain and normal coronary arteries. Eur J Pain 2007:11:360-5. doi:10.1016/i.ejpain.2006.04.002

95 Luo C, Liu D, Wu G, et al. Effect of enhanced external counterpulsation on coronary slow flow and its relation with endothelial function and inflammation: a mid-term follow-up study. Cardiology 2012;122:260-8. doi:10.1159/000339876

96 Masumoto A, Mohri M, Shimokawa H, Urakami L, Usui M, Takeshita A. Suppression of coronary artery spasm by the Rho-kinase inhibitor fasudil in patients with vasospastic angina. Circulation 2002;105:1545-7. doi:10.1161/hc1002.105938

97 Mohri M, Shimokawa H, Hirakawa Y, Masumoto A, Takeshita A. Rho-kinase inhibition with intracoronary fasudil prevents myocardial ischemia in patients with coronary microvascular spasm. J Am Coll Cardiol 2003;41:15-9. doi:10.1016/S0735-1097(02)02632-3
98 Kissel CK, Nikoletou D. Cardiac Rehabilitation and Exercise Prescription in Symptomatic Patients with Non-Obstructive Coronary Artery Disease-a Systematic Review. Curr Treat Options Cardiovasc Med 2018;20:78. doi:10.1007/s11936-018-0667-2

99 Yoshida K, Inoue T, Hirakawa N, Node K. Endoscopic thoracic sympathectomy as a novel strategy for vasospastic angina refractory to medical treatments. J Cardiol 2008;52:49-52. doi:10.1016/j. jjcc.2008.04.002

100 Kronhaus KD, Lawson WE. Enhanced external counterpulsation is an effective treatment for Syndrome X. Int J Cardiol 2009;135:256-7. doi:10.1016/j.ijcard.2008.03.022

101 Giannini F, Baldetti L, lelasi A, et al. First experience with the coronary sinus reducer system for the management of refractory angina in patients without obstructive coronary artery disease. JACC CardiovasC Interv 2017;10:1901-3. doi:10.1016/j.jcin.2017.06.062

102 Beltrame JF. Advances in understanding the mechanisms of angina pectoris in cardiac syndrome X. Eur Heart / 2005;26:946-8. doi:10.1093/eurheartj/ehi242

103 Inaba Y, Chen JA, Bergmann SR. Quantity of viable myocardium required to improve survival with revascularization in patients with ischemic cardiomyopathy: A meta-analysis. J Nucl Cardiol 2010;17:646-54. doi:10.1007/s12350-010-9226-2

104 Cannon RO3rd, Quyyumi AA, Mincemoyer R, et al. Imipramine in patients with chest pain despite normal coronary angiograms. N Eng J Med 1994;330:1411-7. doi:10.1056/NEJM199405193302003

105 Colquhoun DM. Electrical neurostimulators for pain relief in angina. Br Heart / 1991;65:234-5. doi:10.1136/hrt.65.4.234-b

106 Sgueglia GA, Sestito A, Spinelli A, et al. Long-term follow-up of patients with cardiac syndrome $X$ treated by spinal cord stimulation. Heart 2007:93:591-7. doi:10.1136/hrt.2006.102194

107 McGillion M, Arthur H, Andréll P, Watt-Watson J. Self management training in refractory angina. BMJ 2008;336:338-9. doi:10.1136/ bmi.39465.522569.80

108 Knuuti J, Wijns W, Saraste A, et al, ESC Scientific Document Group. 2019 ESC Guidelines for the diagnosis and management of chronic coronary syndromes. Eur Heart / 2020;41:407-77.

109 Beltrame JF. Prinzmetal's Variant Angina: A Contemporary Perspective. In: Braunwald E, ed. Harrison's Advances in Cardiology. 1st ed. McGraw-Hill, 2003: 294-302.

110 Safdar B, D’Onofrio G, Dziura J, Russell RR, Johnson C, Sinusas AJ. Prevalence and characteristics of coronary microvascula dysfunction among chest pain patients in the emergency department. Eur Heart J Acute Cardiovasc Care 2020;9:5-13. doi:10.1177/2048872618764418

111 Yildirim AB, Basarici I, Kucuk M. Recurrent ventricular arrhythmias and myocardial infarctions associated with cocaine induced reversible coronary vasospasm. Cardiol / 2010;17:512-7.

112 Chen JP. Methamphetamine-associated acute myocardial infarction and cardiogenic shock with normal coronary arteries: refractory global coronary microvascular spasm. / Invasive Cardiol 2007;19:E89-92. doi:10.1016/S0167-5273(08)70628-4

113 Bassi S, Rittoo D. Ecstacy and chest pain due to coronary artery spasm. Int J Cardiol 2005;99:485-7. doi:10.1016/i. ijcard.2003.11.057

114 Janjua M, Badshah A, Allen SA. Images in cardiology. Epinephrineinduced ST elevation: a case of endobronchial topical epinephrineinduced coronary vasospasm. Heart 2009;95:656. doi:10.1136/ hrt.2008.161646

115 Baumeister TB, Wickenbrock I, Perings CA. STEMI secondary to coronary vasospasm: possible adverse event of methylphenidate in a 21-year-old man with ADHD. Drug Saf Case Rep 2016;3:10. doi:10.1007/s40800-016-0035-7

116 Robertson RM, Wood AJ, Vaughn WK, Robertson D. Exacerbation of vasotonic angina pectoris by propranolol. Circulation 1982;65:2815. doi:10.1161/01.CIR.65.2.281

117 Song JK, Park SW, Kang DH, et al Safety and clinical impact of ergonovine stress echocardiography for diagnosis of coronary vasospasm. J Am Coll Cardiol 2000;35:1850-6. doi:10.1016/ S0735-1097(00)00646-X

118 Mongiardo R, Finocchiaro ML, Beltrame J, et al. Low incidence of serotonin-induced occlusive coronary artery spasm in patients with recent myocardial infarction. Am J Cardiol 1996;78:84-7. doi:10.1016/S0002-9149(96)00230-5

119 Shimizu M, Hata K, Takaoka H, et al. Sumatriptan provokes coronary artery spasm in patients with variant angina: possible involvement of serotonin 1B receptor. Int J Cardiol 2007;114:188-94. doi:10.1016/j.ijcard.2006.01.026

120 Santoro F, Spennati G, leva R, et al. Late onset of coronary vasospasm after administration of methyl-ergometrine for gynecologic bleeding Int / Cardiol 2012:161:e29-30 doi:10.1016/jijcard 2012.03.156

121 Acikel S, Dogan M, Sari M, Kilic H, Akdemir R. Prinzmetal-variant angina in a patient using zolmitriptan and citalopram. Am J Emerg Med 2010;28:257. doi:10.1016/j.ajem.2009.05.021

122 Maseri A, Lanza G. Fluorouracil-induced coronary artery spasm. Am J Med 2001;111:326-7. doi:10.1016/S0002-9343(01)00930-5 
123 Goldsmith YB, Roistacher N, Baum MS. Capecitabine-induced coronary vasospasm. J Clin Oncol 2008;26:3802-4. doi:10.1200/ CO.2008.17.1637

124 Porto I, Leo A, Miele L, Pompili M, Landolfi R, Crea F. A case of varian angina in a patient under chronic treatment with sorafenib. Nat Rev Clin Oncol 2010:7:476-80 doi:10.1038/nrclinonc.2010.67

125 Sidi A, Dahleen L, Gaspardone A. Coronary vasospasm during anesthesia induction: awareness, recognition, possible mechanisms, anesthetic factors, and treatment. / Clin Anesth 2008;20:64-9. doi:10.1016/j.jclinane.2007.02.016

126 Yeung AC, Vekshtein VI, Krantz DS, et al. The effect of atherosclerosis on the vasomotor response of coronary arteries to mental stress. N Engl J Med 1991;325:1551-6. doi:10.1056/ NEJM199111283252205

127 Chauhan A, Mullins PA, Taylor G, Petch MC, Schofield PM. Effect of hyperventilation and mental stress on coronary blood flow in syndrome X. Br Heart J 1993;69:516-24. doi:10.1136/hrt.69.6.516

128 Arrighi JA, Burg M, Cohen IS, et al. Myocardial blood-flow response during mental stress in patients with coronary artery disease. Lancet 2000;356:310-1. doi:10.1016/S0140-6736(00)02510-1

129 Yoshida K, Utsunomiya T, Morooka T, et al. Mental stress test is an effective inducer of vasospastic angina pectoris: comparison with cold pressor, hyperventilation and master two-step exercise test. Int J Cardiol 1999;70:155-63. doi:10.1016/S0167-5273(99)00079-0

130 Rutledge T, Reis SE, Olson M, et al, Women's Ischemia Syndrome Evaluation (WISE). History of anxiety disorders is associated with a decreased likelihood of angiographic coronary artery disease in women with chest pain: the WISE study. I Am Coll Cardiol 2001:37:780-5 doi:10.1016/S0735-1097(00)01163-3

131 Katon W, Hall ML, Russo J, et al. Chest pain: relationship of psychiatric illness to coronary arteriographic results. Am J Med 1988;84:1-9. doi:10.1016/0002-9343(88)90001-0

132 Asbury EA, Creed F, Collins P. Distinct psychosocial differences between women with coronary heart disease and cardiac syndrome X. Eur Heart/ 2004;25:1695-701. doi:10.1016/j.ehj.2004.07.035

133 Bass C, Wade C, Hand D, Jackson G. Patients with angina with normal and near normal coronary arteries: clinical and psychosocial state 12 months after angiography. Br Med I (Clin Res Ed) 1983;287:1505-8. doi:10.1136/bmi.287.6404.1505

134 Takahashi J, Nihei T, Takagi Y, et al, Japanese Coronary Spasm Association. Prognostic impact of chronic nitrate therapy in patients with vasospastic angina: multicentre registry study of the Japanese coronary spasm association. Eur Heart J 2015;36:228-37. doi:10.1093/eurheartj/ehu313

135 Meune C, Joly LM, Chiche JD, et al. Diagnosis and management of out-of-hospital cardiac arrest secondary to coronary artery spasm. Resuscitation 2003;58:145-52. doi:10.1016/S03009572(03)00149-7

136 Meisel SR, Mazur A, Chetboun I, et al. Usefulness of implantable cardioverter-defibrillators in refractory variant angina pectoris complicated by ventricular fibrillation in patients with angiographically normal coronary arteries. Am J Cardiol 2002;89:1114-6. doi:10.1016/S0002-9149(02)02283-X

137 Chevalier P, Dacosta A, Defaye P, et al. Arrhythmic cardiac arrest due to isolated coronary artery spasm: long-term outcome of seven resuscitated patients. J Am Coll Cardiol 1998;31:57-61. doi:10.1016/S0735-1097(97)00442-7

138 Handberg EM, Merz CNB, Cooper-Dehoff RM, et al. Rationale and design of the Women's Ischemia Trial to Reduce Events in Nonobstructive CAD (WARRIOR) trial. Am Heart J 2021;237:90-103. doi:10.1016/j.ahj.2021.03.011

139 Arnold SV, Grodzinsky A, Gosch KL, et al. Predictors of physician under-recognition of angina in outpatients with stable coronary artery disease. Circ Cardiovasc Qual Outcomes 2016;9:554-9. doi:10.1161/CIRCOUTCOMES.116.002781

140 Phaneuf DC, Waters DD, Dauwe F, Théroux P, Pelletier G, Mizgala HF. Refractory variant angina controlled with combined drug therapy in a patient with a single coronary artery. Cathet Cardiovasc Diagn 1980;6:413-21. doi:10.1002/ccd.1810060410

141 Maseri A. Variant angina. In: Ischemic heart disease. A rational basis for clinical practise and clinical research. Churchill Livingstone; 1995:559-88.

142 Ma YG, Wang JW, Bai YG, Liu M, Xie MJ, Dai ZJ. Salidroside contributes to reducing blood pressure and alleviating cerebrovascular contractile activity in diabetic Goto-Kakizaki rats by inhibition of L-type calcium channel in smooth muscle cells. BMC Pharmacol Toxicol 2017:18:30 doi:10.1186/s40360-017-0135-8

143 Henry TD, Losordo DW, Traverse JH, et al. Autologous CD34+ cell therapy improves exercise capacity, angina frequency and reduces mortality in no-option refractory angina: a patient-level pooled analysis of randomized double-blinded trials. Eur Heart J 2018;39:2208-16. doi:10.1093/eurheartj/ehx764

144 Rai B, Shukla J, Henry TD, Quesada O. Angiogenic CD34 stem cell therapy in coronary microvascular repair-a systematic review. Cells 2021;10:1137. doi:10.3390/cells10051137

145 Verheye S, Jolicœur EM, Behan MW, et al. Efficacy of a device to narrow the coronary sinus in refractory angina. N Engl J Med 2015;372:519-27. doi:10.1056/NEJMoa1402556

146 Giannini F, Baldetti L, Ponticelli F, et al. Coronary Sinus Reducer Implantation for the Treatment of Chronic Refractory Angina: A Single-Center Experience. JACC Cardiovasc Interv 2018;11:784-92. doi:10.1016/j.jcin.2018.01.251

147 Kunadian V, Chieffo A, Camici PG, et al. An EAPCI Expert Consensus Document on Ischaemia with Non-Obstructive Coronary Arteries in Collaboration with European Society of Cardiology Working Group on Coronary Pathophysiology \& Microcirculation Endorsed by Coronary Vasomotor Disorders International Study Group. Eur Heart 2020;41:3504-20. doi:10.1093/eurheartj/ehaa503

148 Ford TJ, Ong P, Sechtem U, et al, COVADIS Study Group. Assessment of Vascular Dysfunction in Patients Without Obstructive Coronary Artery Disease: Why, How, and When. JACC Cardiovasc Interv 2020;13:1847-64. doi:10.1016/j.jcin.2020.05.052 